\title{
GERHARD WirTh
}

\section{Der Kaiser und der Untertan}

\author{
Erwägungen zu den inneren Schwierigkeiten des römischen Imperiums*
}

Die Alte Geschichte scheint ihre Bedeutung als zentrales Fach im modernen Bildungsgefüge in den letzten Jahren weitgehend verloren zu haben. Freilich, teilt sie dieses Schicksal mit dem der Altertumswissenschaft schlechthin, so war die Verbindung zur Geschichte mit deren verschiedenen Zweigen doch nie so eng, daß von hier aus eine Wiederbelebung zu erwarten wäre. Und ob die Rolle als Metier der Unterhaltung für ein Publikum eine Zukunft bedeutet, das nicht mehr verlangt, bleibt abzuwarten, dies gilt am spektakulärsten gerade für die Länder, in denen sie wirklich eine Tradition hat und demnach man den Verlust am stärksten spürt. Einschlägige Veranstaltungen der Universitäten registrieren sinkende Zahlen an Interessierten über das notwendige Soll von Pflichtkursen hinaus, Stimmen zur Überflüssigkeit entsprechender Studien in einer allzu gegenwartsbezogenen Umgebung sind ebenso häufig und penetrant wie die, die das Gegenteil verkünden. Die Gründe für das alles sind vielfältig. Daß sie allein an der Hochschule selbst zu suchen seien, ist eine besonders gefährliche, weil unzutreffende Behauptung, obzwar der Stellenwert entsprechender Studien - und nicht nur dieser - deutlich sinkt, weil sich

* Die Arbeit versucht, die wirtschaftlichen und die sozialen Kriterien der römischen Kaiserzeit und der Spätantike auf einen gemeinsamen Nenner zu bringen. Geschehen kann dies nur von den offenkundig wichtigsten, auffallendsten Symptomen her. An ein umfassendes, alles enthaltendes Gesamtbild ist nicht gedacht, ein solches wäre unmöglich. Dabei erweist sich der im Verlauf der Jahrhunderte scheinbar entwickelnde Zwangsstaat als die Folge eines Bemühens, in der Sicherung der politischen und nicht zuletzt der physischen Fortexistenz, es allen Beteiligten recht zu machen und von seiten des Gesetzgebers her ein Gleichgewicht aufrecht zu erhalten. Den zwangsläufig sich ergebenden Belastungen konnte auch die Kirche nicht gerecht werden. Colonat, Collegia und andere Institutionen solcher Art haben die Funktion einer Sicherung des Bestehenden, während die Aufgabe der oberen Schichten bzw. der Aristokratie es ist, dem Staat Aufgaben abzunehmen.

Im folgenden geht es nicht um ein geschlossenes Bild. Deren gibt es bereits allzu viele. Sondern es sind einzelne Fragen, die gleichsam zwischen scheinbar unverrückbaren, allzu gut dokumentierten Erkenntnissen liegen oder aus solchen sich herauskristallisieren lassen, und um deren Hintergründe, die man gerne übersieht. Die Einleitung hat mit all dem auf den ersten Blick wenig zu tun. Sie erklärt sich indes aus der Sorge, daß nunmehr stärker als je zuvor die Grundlagen drohen verloren zu gehen für eine Beschäftigung mit dem, was hier zur Sprache kommt, und diese selbst damit einfach ihren Sinn verliert. Und all dies, ohne daß für den Verlust ein humanitärer oder selbst auch nur anthropologisch verwendbarer Ersatz vorerst sichtbar wäre. Auf Fußnoten wurde verzichtet, sie würden, so verwendet, wie es vielleicht nötig wäre, jeden Rahmen sprengen. Eine Liste sachdienlicher oder sogar weiterführender Sekundärliteratur wird beigefügt. Daß auch sie nur Auswahl und Stückwerk sein kann, versteht sich von selbst.

In seinem Kern sollte das Vorliegende Bestandteil einer Festschrift für K. Hoheisel werden. Eine lange Krankheit hat die Fertigstellung über Gebühr verzögert. Doch ist der Österreichischen Akademie der Wissenschaften zu danken, daß sie eine Gratulation dennoch ermöglicht. 
die Mühe, die man aufzuwenden hat, nicht mehr lohne. Es ist denn auch gleichgültig, ob es der Staat ist, der die entsprechenden Institutionen finanziert und dabei seine eigenen Interessen verwirklicht - solche haben im Laufe der Wissenschafts- wie Geistes- und Ideengeschichte stets gewechselt - oder aber private Träger, denen es notgedrungen stets um Gewinn ging und deren Existenz ohne entsprechende Anpassung noch weniger denkbar war.

Man darf im übrigen das Bild aber doch nicht gleich allzu schwarz malen. In jedem Semester findet sich eine Zahl von Studierenden, die sich zu gewisser Vorliebe gerade für diesen Abschnitt der Menschheitsgeschichte durchringen, welche mit der Völkerwanderung zu Ende geht, und die Anregungen dazu, man möchte staunen über solche Ausnahmen von der Norm, bereits in der Schule gefunden haben. Sie mögen sich schwerer tun als ihre Vorgänger vor zwanzig oder selbst noch vor zehn Jahren, denn es ist ihnen nur mit größter Mühe noch möglich, sich die Voraussetzungen zu erwerben. Was sie nach Überwindung derartiger unverschuldeter Anfangsschwierigkeiten beizubringen in der Lage sind, indes erweist sich sehr häufig als kaum weniger gehaltvoll im Vergleich zu dem, was unter gleicher Bedingung jene früheren Generationen leisteten. Und die Klage über einen allgemeinen Verfall scheint auf den ersten Blick vorerst unberechtigt. Die Sache ist sich gleich geblieben daß der Mensch ein anderer ist, um den es dabei jetzt geht, tut wenig zu unserer Sache. Die Frage freilich liegt eher in der Breite, in der Zahl derer, die, mit entsprechenden Ansätzen allgemeiner Bildung ausgestattet, eine allgemein gültige Wirklichkeit zu deuten vermögen und dabei zu jener Rechenschaft von dreitausend Jahren in der Lage sind, oder nach einer solchen suchen wollen. Und dies ist es, was in der Tat zu denken gibt. Dabei mag sich die Frage nach der Alten Geschichte relativieren, bleibt mit dem scheinbar Spezifischen doch auch an Übergreifendem, Allgemeinem allzu viel im Unwägbaren, Undurchdringlichen, und wird das Studium oder selbst das Bemühen um sie gerade deshalb sogar doppelt gefährlich. Nicht, daß es hier um ein Hochhalten esoterischer Prinzipien ginge. Der Wert einer Wissenschaft läßt sich allein weder an der Zahl erscheinender Dissertationen und Aufsätze, fachwirksamer Neuerkenntnisse und Entdeckungen und schon gar nicht an der Zahl verfügbarer Hochschulsinekuren bemessen. Auch nicht einmal nach dem Nutzen, den sie für die Öffentlichkeit zu erbringen scheint, was immer diese Öffentlichkeit noch mit ihr anzufangen vermag.

Von Bedeutung indes scheint mir, wieviele solcher Studenten alljährlich in den Schuldienst gehen. Denn sie sind es, durch deren Möglichkeit und von da ausgehend überhaupt das Engagement im rein Menschlichen die ersten Grundlagen für eine solche Tradition und deren Fortleben gelegt werden, Grundlagen übrigens, die in ihrer Wirkung sich subjektiv am wenigsten voraussagen lassen und auch durch keine Lehrplanregulierung von vornherein festzulegen sind. Die Art freilich, wie in die letzten und neuesten Lehrpläne der Länder Mitteleuropas fast überall das Fach selbst eingebaut ist, gibt zu denken. Nichts gegen heilsame Beschränkungen und notwendige Akzentverlagerungen, die der Fachidiot am wenigsten zu beurteilen vermag. Aber man wird das Gefühl nicht los, daß gerade dieses begrenzte Feld historischer Erkenntnisvermittlung in einer besonderen Gefahr ist, einem Simplifizierungsprozeß zum Opfer zu fallen, dem eine unverkennbare Fehldeutung zugrunde liegt. Freilich, der Einbau in die Bildungspolitik ist seit je das Ende nicht nur von Ideengeschichte in Teilen oder im Ganzen gewesen, und zwar mit allem, was dazu gehört. Das aber heißt, letztlich nicht nur der Forschung, sondern dazu jeder Bildung als solcher, und für unsaubere Manipulationen ergab sich 
dabei stets ein weites Feld, wenn es darum ging, die Funktionen des Multiplikators zu verschieben. Jede Epoche letztlich schafft sich die Menschen mit den Qualitäten, die sie braucht, und für die Interessen dieser Menschen gilt das gleiche. Abzuwarten, was die Folgen dieses Abkürzens denn sein werden, scheint mir besonders gefährlich, denn bis hier die wirklich brauchbaren Erfahrungen zu gewinnen sind, könnte es zu spät sein. Nicht daß es darum ginge, nun mit allem Eifer die Möglichkeit instruktiver moderner Analogien zu den Lieben Alten und deren Verwendbarkeit aufzuzeigen. Man hat das leider schon zu oft getan, der Gesamteindruck ist der reine Ausverkauf, an dem sich seit einiger Zeit auch die erlauchtesten Geister des Faches selbst mit beteiligen. Die Frage, was dem modernen Menschen das Altertum und dessen Geschichte zu sagen habe, jenes Wechselverhältnis zwischen entferntester faßbarer Vergangenheit und Gegenwart, scheint diffiziler zu betrachten. Aber schon der Versuch einer Antwort vielleicht hilft weiter als sich dies auf den ersten Blick erkennen läßt.

Geht man von der für uns so charakteristisch gewordenen und gerne geübten Einbettung historischen Geschehens in wirtschaftliche und soziale Gefüge aus, so wäre festzustellen, daß etwa auch das römische Imperium ohne diese nicht denkbar ist, so daß es mit seinen Problemen schon deshalb zeitlos an Charakter gewinnt. Die Kaiserzeit etwa als bewußter Neuanfang aus einer deutlich sichtbaren Krise in allen Bereichen, nicht nur der res publica, würde ohne entsprechende Erwägungen vollends unverständlich bleiben. Und dies nicht einmal so sehr von Gedanken aus, wie sie sich aus der geographischen Lage der alten Welt eigentlich ohne weiteres ergeben müßte, hat das Imperium mit seinen Belangen mit Rom selbst, der Hauptstadt, an sich stets doch wenig zu tun. Man wünscht sich aus den Quellen mehr darüber, wie sich die Synthese vollzog. Wie wenig dies der Fall ist, hat schon Rostovtzeff gezeigt. Römische Wirtschafts- wie auch Außen- und Imperiumspolitik sind vielmehr durch eine Richtungslosigkeit gekennzeichnet, die auffällt und auch bei wohlwollender, differenzierter Betrachtung nur schwer zu begreifen ist, von Kausalitäten abgesehen, die schon angesichts ihrer Vordergründigkeit nicht weiter helfen können, aber die zwangsläufig jetzt ihre Gültigkeit haben. Man paßt sich an. Bleibt diese Anpassung aber Provisorium, so erklärt sich eine allgemeine Verschlechterung des Zustandes zwischen Kaiserzeit und Ende der Antike aus den äußeren wie inneren bekannten Voraussetzungen zur Genüge. Ursachen und Wirkung sind scheinbar eindeutig.

Doch eine Monokausalität läßt sich in der ganzen Weltgeschichte kaum an einer Stelle weniger sichtbar machen. Wir selbst freilich laufen allzuleicht Gefahr, wie fast in allen Bereichen so auch hier, die eigene Gegenwart allzu sehr auf die Vergangenheit zu übertragen und uns einfach die entsprechenden Termini so zu deuten, wie wir sie verstehen. Nein, worum es diesem Imperium geht, gehen muß, ist Primitiveres, Wichtigeres - es ist im weitesten Sinne des Begriffes die Versorgung der Reichsbewohner, die zu allen Zeiten eine prekäre war. Bei all dem soll hier auch nicht der Wasserkopf Rom zur Sprache kommen, der immer eine Ausnahme darstellte, überdies seit Caesar vieles an seiner Wertigkeit in solchen Dingen verlor und wenig Charakteristisches mehr an sich hat - östliche Städte von kaum geringerer Bevölkerungszahl zeigen, daß die spezifisch römischen Probleme keineswegs allgemein verbindlich gewesen sein müssen. Doch es bleibt zu fragen, und dies bei all unseren Kenntnissen zur Republik und deren Expansion, wie weit schon damals entsprechende Erwägun- 
gen die römische Politik mit bestimmt hatten. Die Auflösung des hellenistischen Durcheinanders von Monarchien ließ sich wohl so erklären, die Folgen einer schwindenden Stabilität sind unseren Quellen noch einigermaßen zu entnehmen. Die Rolle des Imperiums müßte demnach lange vor Augustus klar gewesen sein. Im übrigen hatte ein offizieller Monopolismus für die hier entscheidenden Fragen in den Reichen längst seine eigene Gesetzmäßigkeit entwickelt. Spenden, Schenkungen von offizieller oder privater Seite und nicht zuletzt von seiten der Herrscher selbst schufen eine Internationalität, die in der hellenistischen Zeit wirkliche Katastrophen verhindert zu haben scheint.

Das Imperium freilich konnte sich mit solchen spontanen Aktionen allein nicht mehr zufrieden geben, obzwar die kaiserliche Munifizenz in Notfällen das Ihre tat, Schlimmstes zu verhindern. Dies gilt auf die ganze Oikumene bezogen, wobei die Voraussetzungen den Gegebenheiten entsprechend von Fall zu Fall sich unterscheiden mögen, die Probleme aber die gleichen bleiben. In der Selbstdarstellung der Antike ist dieser Bereich des Lebens zwar nur ein Ausschnitt, und er erleichtert unsere Erkenntnisse kaum, so daß wir im wesentlichen weniger auf Indizien als auf indirekte Schlußfolgerungen angewiesen sind. Aber auch auf diese Weise läßt sich aus dem Schweigen unserer wichtigen Quellen über Konkretes, vordringlich Notwendiges noch einiges entnehmen, das weiter hilft.

Die Romdeutung, die von der ethischen Rolle für den ganzen orbis terrarum ausgeht, hat mit dem, was hier angedeutet werden soll, nichts zu tun. Eher im Gegenteil. Zeitlich bedingte Unterschiede sind bei all dem von geringem Belang, denn die allgemeinen Bedingungen bleiben die gleichen bis ans Ende. Und Entsprechendes gilt für die räumlichen Differenzen, auch wenn die Voraussetzungen im Osten andere waren als im Westen, was denn auch für die Folgen gilt. So zeigt eine große Zahl von Nachrichten, daß etwa in Ägypten trotz Pharaonen und Ptolemäern mit ihrer Verbindung von ökonomischen, politischen und wirtschaftlichen Interessen eine wirkliche Intensivbewirtschaftung des Bodens erst in der Kaiserzeit begann, und ähnliches wird trotz des karthagischen Vorbildes in einem großen Rahmen für Afrika gelten. Nein, zu fragen ist, wie Politik und Wirtschaft sich miteinander überhaupt in ein Wechselverhältnis bringen lassen und welche anderen Folgen staatlichen Bemühens um Stabilität sie durch solche Eingriffe notwendig dann gezeitigt haben. Auch die Frage nach dem Selbstverständnis des Kaisertums spielt in diesem Zusammenhang kaum eine Rolle. Ein solches scheint trotz ideologischen Sichentwickelns vom Prinzipat zum Dominat zementiert und mit einem ethischen Hintergrund versehen, der sich aus griechischer Wurzel herleitet und keinen Augenblick in Zweifel stand. Die Terminologie jedenfalls geht nahtlos ineinander über: Panegyrik, Rhetorik und Inschriften geben uns ein wenn nicht klares, doch umfasssendes Bild.

Daß sich das Kaisertum hier seiner Aufgaben bewußt war, folgt dabei eigentlich schon aus der Geschichte seines Entstehens. Nicht zuletzt deshalb bedeuten die einschlägigen Maßnahmen, die es von Fall zu Fall ergreift, niemals vordergründige Proletarierversorgung im gracchischen Sinn auf engem Raum - derartiges ist längst vorbei. Vielmehr handelt es sich bei entsprechender Vorstellung von Wohlfahrt stets um nicht weniger als um die Sicherung der Lebensbedingungen schlechthin und damit um die Ernährungsgrundlage auf Dauer für ein ganzes Weltreich mit Bedingungen, deren Vielfalt seinen Dimensionen entspricht. D. h., was verbindet, sind Bodennutzung und Landwirtschaft, deren Stabilisierung ohne eine Planung auf Dauer gar nicht denkbar ist. Und all dies ist nicht mehr auf eine einzelne, privilegierte 
Stadt zu beziehen, sondern zumindest als Postulat allgemeinen Charakters zu sehen. Dazu kommen die Digesten als Rechtsgutachten dem Kaiser nahestehender Juristen. Sie lassen sich in ihrer Mehrzahl doch nur mit entsprechenden Fragen in Zusammenhang bringen und von ihnen heraus erst verstehen. Für die Bodenbewirtschaftung selbst freilich sind wir zudem nicht ganz ohne andere Hinweise, die weiter helfen können. Sie betreffen zwar im wesentlichen wohl Italien und stammen aus früherer Zeit. Sie kennzeichnen aber gut den allgemeinen Übergang aus der Republik und die beginnende Ausdehnung zum Kaiserreich. Es sind dies Catos Buch De Agricultura, Varros De re Rustica und Columellas Werk von der Landwirtschaft. Von ihnen scheint der erste gleichsam aus dem Vollen zu schöpfen. Landwirtschaft wird in kapitalistischer Absicht betrieben, der Anbau ist von Erwägungen des Nutzens und der Rentabilität der Verkaufsmöglichkeiten bestimmt, zu all dem steht ein offensichtlich unerschöpfliches Sklavenreservoir zur Verfügung, mit dem man dementsprechend verfährt. Alle weitere Entwicklung aber von da aus ist die Anpassung an veränderte Umstände: Varro bereits scheint in anderer Weise rational zu denken. Nicht nur daß er die bereits weitgehend offensichtlich vorwiegend als Hobby in Italien betriebene Weidewirtschaft abzubauen sucht: War für Cato die Frage der Intensivierung des Anbaus angesichts anderweitiger Versorgungsmöglichkeiten leicht zu lösen und ging in seinen Erwägungen gleichsam nebenher, jetzt wird die Erzeugung von Grundnahrungsmitteln in größtem Rahmen als vordringlich betont, wirkt aber zugleich schon wie ein wachsender Zweifel an entsprechenden Möglichkeiten für das eben im Entstehen begriffene Imperium, dessen Zukunft dem Verfasser in der Tat fragwürdig war. Mit Jagd- oder Weidewirtschaft allein ist eine solche Stabilität in der Lebensgrundlage aber nun einmal nicht mehr zu gewährleisten. Zugleich tritt schon der Gedanke einer Autarkie der Gutswirtschaft in den Vordergrund. Die Entwicklung zum Regionalismus und die Intensivierung der Versorgung auf engstem Raume, das sind die Anfänge, mit denen sich der Prinzipat demnach auseinanderzusetzen hat. Bei Columella dann ist die Entwicklung, die sich hier abzeichnet, vollendet. Bei ihm schon zeichnen sich die Probleme ab, die später sich zuspitzen.

Mir scheint, daß bereits aus solchen Zeugnissen die eigentliche Imperiumskrise früh deutlich wird, und dies, obzwar unsere literarische oder aber die juristische Überlieferung nie ganz klar zwischen Rom, Italien und dem ganzen Imperium unterscheidet. In der Tat, Gemeinsamkeiten sind unverkennbar. Denn all diese Erwägungen lassen sich (und dies besonders in Beziehung zu anderen Symptomen) am ehesten aus einer Erschöpfung der gesamten antiken Welt erklären, die auf diese Weise den grandiosen Ablauf griechischer und römischer Geschichte mit Entvölkerung, Brachliegen und Kräftemangel zu bezahlen hat, Symptomen, wie sie analog in der Weltgeschichte fast niemals ausgeblieben sind. Daß dies nicht nur für die Städte sondern für das flache Land gilt, versteht sich von selbst, fast überall haben archäologische Forschungen ergeben, daß die Blüte unter der Herrschaft Roms gerade aus solchen Gründen nur eine kurze gewesen sein kann. Die offizielle Reaktion setzt denn früh ein. Seien wir mit den Jahrhunderten nicht kleinlich: An die Ehe- und Bevölkerungsgesetzgebung eines Augustus schließt sich die Alimentarorganisation der Adoptivkaiser. All dies zwar scheint auf Italien bezogen, aber ein Modellcharakter ist unverkennbar. Ich frage mich, ob nicht die 
Germanen- und Dakienkriege Domitians oder Traians nicht zuletzt mit von der Bemühung um einen neuen Zugang zu den wichtigsten Menschenreservoiren ihrer Zeit bestimmt waren, Zeugnisse von Statthaltern an der Grenze gibt es denn genug schon im 1. Jhdt, die sich rühmen, Barbaren im Imperium aufgenommen zu haben. Die Zahlen, die dabei genannt werden, verblüffen, aber an einem offiziellen Hintergrund der Inschriften wie an der Absicht ihrer Publikation ist kaum zu zweifeln. Mark Aurel jedenfalls zieht die Konsequenz in dieser Form und siedelt Markomannen in riesiger Zahl in Italien an, das demnach um die Mitte des 2. Jhdts. weitgehend entvölkert gewesen sein und viel Platz geboten haben muß. Die Notwendigkeit, intensiv nach neuen Möglichkeiten von Versorgung und Ernährung zu suchen, Ernährungsfragen einer- und Bevölkerungs- bzw. Arbeitskräftemangel anderseits, das scheint der Nenner, auf dem man die Politik, selbst die auswärtige, auch unter den besten Kaisern zu bringen hat, soweit sie sich auf das ganze Imperium beziehen.

Die Gründe wurden immer wieder erörtert, zu einem eindeutigen Ergebnis freilich wird nie zu kommen sein. Kriegs-, Militär-, Verwaltungs- und selbst Geistesgeschichte aber scheint von dem hieraus resultierenden, die Dinge begleitenden Angstgefühl mitbestimmt, und das Lavieren zwischen Notwendigkeit und Wirklichkeit ist in keinem Bereich zu übersehen. Ein weiteres kommt hinzu: Wohl war das Imperium in seinen besten Zeiten ohne Mühe in der Lage gewesen, mit seinen Einnahmen finanzielle Verpflichtungen zu erfüllen, und hielten Einnahmen wie Ausgaben einander die Waage. Seit erwähnter Zeit muß das Gefüge solcher Verpflichtungen zu derartiger Belastung geführt haben, daß man nicht mehr umhin konnte, zur erhöhten Besteuerung zu schreiten und diese ständig zu verschärfen. Damit aber wird das Finanz- zum Wirtschaftsproblem. Denn besteuern ließ sich, neben stets fluktuierendem, in seinem Gewinn aber nie ganz berechenbarem Handel und der Produktion von Waren als fixes Objekt nur der genutzte Boden, und naturgemäß hat demnach die Nahrungs- zugleich auch als Geldquelle herzuhalten. So ist es denn wohl verständlich, daß gerade aus dieser Zeit in den Digesten erstmals etwas sichtbar wird wie eine offizielle, kaiserliche Fürsorge vor allem für die landwirtschaftliche Infrastruktur, d. h. für die Elemente, die so in erster Linie mit einer Doppelaufgabe belastet waren.

Es wäre wohl falsch, dabei lediglich von geregelter Ausbeutung oder den Möglichkeiten einer Ausnutzung menschlicher Kräfte bis zur physischen oder finanziellen Belastbarkeit zu sprechen, obwohl es den Anschein hat, es sei um eine solche gegangen. Indes, was konnte die politische Führung Roms unter gegebenen Umständen, ohne umfassende wirtschaftliche Konzeptionen und Modelle und auch ohne wirkliche Erfahrungen, anderes tun, um mit dem fertigzuwerden, was sich seit Hadrian immer deutlicher als eine allgemeine Katastrophe abzeichnete? Was bleibt, ist die Strapazierung vorhandener Modelle, nicht mehr. Formen solcher Ausnutzung der Infrastruktur des Versorgungssektors sind alt und haben Vorläufer in der griechischen, besonders in der hellenistischen Geschichte. So läßt sich der Colonat, als die Verpachtung des Großbesitzes zu besserer Nutzung, bereits im hellenistischen Ägypten nachweisen: Hier wie dort verbleibt dem Pächter ein großer Teil des Erwirtschafteten zur eigenen Verwendung. Pacht aber ist verwandt mit der Miete. Verbindet man Zeugnisse beider Sektoren miteinander, so läßt sich ein geradezu brennendes, auch den modernen Erkenntnissen entsprechendes Interesse nicht übersehen, schon um der Effektivität willen die Pachtfreudigkeit zu fördern, Anreize zu eigener Initiative zu geben und vor allem, die Pachtenden möglichst lange an ihrer Stelle zu belassen. Daß Rom dabei auf hellenistische, 
ja auf altorientalische Erfahrungen zurückgreift und aus historischer Kontinuität Nutzen zieht, ist interessant und insofern von Bedeutung, als auf diese Weise östliche Strukturformen der Staatswirtschaft gleichsam künstlich in den Westen mit seinen so ganz anderen Voraussetzungen übertragen scheinen. Vorausgegangen sein müssen all dem freilich Erwägungen zur Effektivität in welchem Zusammenhang auch immer.

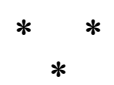

Der Colonat ist auf Privatland wie auf den kaiserlichen Domänen möglich: Die Unterschiede können nur graduelle gewesen sein, zieht doch die kaiserliche Kasse mit der Zeit die Kontrolle der gesamten Reichseinkünfte und damit des gesamten wirtschaftlichen Potentials an sich, d. h. auch dessen, das den eigentlich privaten Sektor ausmacht, und bestehen Möglichkeiten, Pachtverhältnisse in beiden Sektoren miteinander zu kombinieren. Daß mit dem Colonat darüber hinaus Hand- und Spanndienste verbunden waren, hat Entrüstungsstürme besonders sozialistischer Historiker hervorgerufen. Indes - auch diese Dienstleistungen scheinen fest normiert, und die einzigen Zeugnisse beruhen darauf, daß Colonen mit Beschwerden hierüber immerhin gelegentlich bis zum Kaiser vordrangen und Recht bekamen, ob allein auf den kaiserlichen Domänen, ist mir fraglich, generelle Unterschiede ließen sich auf Dauer wohl kaum aufrechthalten. Offizielle Differenzierung innerhalb des Typs selbst und Spezifizierung kann sich ebenfalls nur zugunsten seiner Mitglieder ausgewirkt haben wie auch so verstanden worden sein.

Dazu aber kommt noch ein anderes: Der Colonatsbegriff entstammt der Wirtschaft. Eine genaue Definition erlauben unsere Quellen nicht, es scheint, er blieb auch für Zeitgenossen amorph. Wer ihn als festen Terminus in die Sphäre des Personen- und Sachenrechts überträgt, vergewaltigt nicht nur die antiken Verhältnisse, sondern setzt sie in ein Licht, in dem er selbst die Wirklichkeit bald nicht mehr zu erkennen vermag. Eine entsprechende Verwischung freilich vermögen, wie Bolkestein bereits beklagte, schon die antiken Quellen und selbst die Gesetzestexte kaum zu vermeiden, was seine Arbeit fast scheitern läßt, und es entsteht der Eindruck, als habe der Gesetzgeber in der Tat den Weg einer rhetorischen Umschreibung nur allzu gerne benutzt, um notfalls den Betroffenen, Eigentümern wie Pächtern, und auch allen anderen, die damit zu tun hatten, die Auslegung zusammen mit der Entscheidung zuzuschieben und dabei Hintertüren offen zu lassen. Die Notwendigkeit, Bestimmungen im Laufe der Jahre zu wiederholen, erklärt sich hieraus wohl mit, und nicht zuletzt auch dann, daß Kommunikation und Information selbst von Beamten und deren officia niemals ganz lückenlos oder aber die Möglichkeit von bewußter Ignoranz und von Unterschleif stets allzu groß waren. Vieles an Gesetzen, Termini und Bestimmungen scheint nicht zueinander zu passen oder sich direkt zu widersprechen, zu erklären am ehesten aus vielfältiger Rücksichtnahme oder Unklarheit im einzelnen. An Verwandtem dazu, das einiges erklärte, freilich fehlt es auch in unseren Tagen nicht. Für die Terminologie zur römischen Außenpolitik gilt Analoges. Die Verfallsbilder, die die Nachwelt zeichnet, wiederum betonen zu sehr den moralischen Aspekt: Vereinzelte Hinweise finden sich wohl bereits in den zeitgenössischen Quellen: Unklar bleibt, wie weit diese Zeitgenossen wirklich die sachlichen Kausalitäten zu durchschauen vermochten. Eine Lösung hat keiner von ihnen parat.

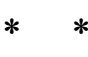

$*$ 
Ich fürchte, das muß betont werden, die Verwischung der Kategorien hat schon zuviel Unheil gestiftet, und dies nicht allein in ideologisch einseitig ausgerichteter Literatur. Denn zum Colonat und zumindest entsprechender Tätigkeit konnten seit je auch Sklaven verwendet werden, und das in der Tat macht die Dinge schwierig. Sklaven in solcher Stellung erscheinen bereits im klassischen Griechenland, das dort Eigentümliche hat hier nicht zu interessieren. Nicht zu übersehen aber ist, daß selbst dort bereits ganz offensichtlich mit der wirtschaftlichen Selbständigkeit und einer gewissen persönlichen Freizügigkeit auch eine offizielle juristische Anerkennung sachlicher Kompetenz sich vermehrt. Der Sklave als Pächter erhielt auffallende Unabhängigkeit in Dingen, die sein Ressort betrafen, ausgedrückt durch einen gewissen staatlichen Schutz in den wirtschaftlichen Belangen. Zwangsläufig aber mußte eine solche zu einem Zustand führen, der den rechtlichen letztlich als unwichtig erscheinen läßt. Wenn man der Terminologie noch weiter nachgehen will - die Verwendung des Sklaven in der kaiserzeitlichen Wirtschaft konnte, konsequent durchgehalten, im Grunde ganz natürlich nur zur allmählichen Auflösung des Phänomens der Sklaverei von innen heraus führen. Sicher, die Abhängigkeit vom Herren, für Sklaven und Colonen, wird stets betont, auch wenn wie in allen Bereichen, die Rechtsquellen eine klare, eindeutige Definition vermeiden und die Terminologie von der Rhetorik bestimmt und deshalb hier merkwürdig schwammig bleibt. Die Digesten heben in Colonatsdingen immerhin aber eine, wenngleich begrenzte Rechtsfähigkeit auch des Sklaven hervor und, genau genommen, scheint man zwischen den verschiedenen Aspekten des Colonats und des Sklavenstandes im Umgang miteinander eine klare Grenze früh nicht mehr erkannt zu haben. Daß Rechtsgutachten offenkundig auch eine solche Grenze immer wieder hervorzuheben scheinen, ist bezeichnend dafür, daß im Sachlichen diese kaum bestand und keiner von denen sich so recht über die juristischen Implikationen im klaren war, die damit zu tun hatten. Dieser Unselbständige kann in eigener Regie pachten und Gewinn für sich erwirtschaften, über den nach Gutdünken zu verfügen dem Herren unendlich schwer gewesen sein muß, was immer die einschlägigen Termine besagten. Damit aber verwässerte sich die an sich sachrechtliche Beziehung von vornherein.

Bereits Varro erwähnt das peculium, das dem Sklaven gehörende Privatvermögen. Sein wichtigster Verwendungszweck wird durch den unter Claudius bereits bekannten fiscus libertatis et peculiorum hinreichend gekennzeichnet, und bei all dem ist wohl nicht zu übersehen, daß es sich dabei um eine staatliche Institution handelte. Wohl erörtern die Juristen häufig den Typ des entlaufenen Sklaven, für den - auch das ist bezeichnend - wohl unter Antoninus Pius oder Mark Aurel sogar eine eigene Polizeiorganisation ins Leben gerufen wurde. Zugleich fällt auf, welche Bedeutung man der freiwilligen Rückkehr dieser Menschen zur Arbeit zuschreibt, sind doch offenkundig schon für einen Columella Sklaven und auch deren Behandlung wichtiger als für Cato. Aber weder um diese Zeit noch später scheint der Colonat eine Institution zur Versklavung Freier gewesen, sondern eher als eine solche zur Befreiung von Sklaven gesehen und sogar gefördert worden zu sein. So ist denn auch nicht zu verwundern, daß schon ein Varro etwa der Ehe von Sklaven innerhalb des eigenen Betriebs besondere Wichtigkeit zumißt, Columella den Wert derartig autogener Möglichkeiten der Personalergänzung besonders lobt und offensichtlich selbst eine eheliche oder eheartige Verbindung von Sklaven und Freien kein besonderes Problem war. Neben einem Abbau der Bestrafungsvorschriften - ein gewisses Hin und Her vielleicht angesichts 
wechselnder allgemeiner Umstände gerade hier wäre vom Historischen her noch zu prüfen - wird das Recht des Colonen betont, sich vor öffentlichem Gericht gegen seinen Herrn zu beschweren. Und als Pendant zu den angedeuteten Möglichkeiten der Rechtsselbständigkeit ja alles in allem fällt in den Digesten geradezu auf, wie wenig eigentlich vom Recht des Herrn noch die Rede ist. Auch von Härte und der oft hervorgehobenen antiken Brutalität der Menschenbehandlung verlautet wenig mehr - ich kann mir dies wie gesagt nur aus der notwendigen Aufweichung im Sachlichen erklären, die früh begann.

Wie sich die Dinge in Wirklichkeit verhielten, freilich bleibt unklar. Zeugnisse für den Abbau entsprechender Unterschiede als Folge philosophischer Postulate für kultivierte Autoren wie Plinius und Seneca indes sind bekannt, und als isoliert oder weltfremd wird man diese nicht betrachten können. Sie werden durch offizielle Schriften und Rechtsgutachten wenigstens indirekt ergänzt. Sicher, die pragmatische Wurzel aller Erwägungen wird dabei nicht zu übersehen sein. Daß indes auf diese Weise lediglich ein Provisorium unternommen wurde, ist kaum zu glauben. Römische Imperiumsdeutung muß sich schon um diese Zeit darüber völlig im klaren gewesen sein, wohin die Dinge führten, die sich deutlich entwickelten, Zeugnisse gibt es genug, und so bleibt m. E. nur der Schluß, daß vieles von dem, was man so gerne als geschichtsimmanent hinstellt, der Gegenstand durchaus rationaler Erwägungen war und bewußt, d. h. unter Erwägung auch der möglichen Konsequenzen, ins Leben gerufen und in ein entsprechendes System zu bringen gesucht wurde. In unserem Fall bedeutet dies, daß man auch da ein Aufgeben überlieferter sozialer Verhältnisse und der zugrundeliegenden Vorstellungen in Kauf zu nehmen bereit war. Der Abbau von Erfahrungen und vielleicht $Z$ wangsvorstellungen aus den Sklavenaufständen und Sklavenkriegen früherer Jahrhunderte kommt hinzu. Solche mögen an allgemeiner Entwicklung einiges verzögert haben, die Kaiserzeit kam um einen bewußten Neuanfang auch in diesen Dingen nicht herum.

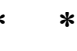

Freilich konnte man auf die besitzenden oberen Schichten der Gesellschaft, zu diesen unteren das natürliche Pendant und seit den frühesten Zeiten das schlechthin tragende Element römischer Politik, auch unter den Kaisern nicht verzichten. Der Begriff ist nicht weit genug zu fassen, soziale Funktionen, politische Aufgaben und allgemeine Rolle unterliegen einem Prozeß von vielfacher Fluktuation, es wäre denkbar, daß erst die späte Antike wirklich lernte, den stabilisierenden Faktor zu erkennen, den diese Oberschicht darstellte. Zu generalisieren freilich verboten unsere Kenntnisse stets, und ebenso gefährlich ist es, das Bekannte auf einzelne Personen von Fall zu Fall zu beziehen. Im Rahmen angedeuteter Entwicklung allerdings tritt diese Oberschicht deutlich zurück. Wohl sahen wir ihre Mitglieder in wichtiger Funktion, und ihr Reichtum nimmt zu, Afrika etwa ist Ende des 1. Jhdts. praktisch in der Hand von sechs oder sieben Familien. Daß es sich dabei lediglich um Großpächter handelt, tut zur Sache wenig. Allgemein, soweit zu erkennen, schränkt man die Handlungsfähigkeit der Grundeigentümer, damit eben zugleich dieser Oberschicht, in Erbfällen fast stets zu Gunsten des nachgeordneten Pächters ein, wenn es zu Streit- oder Definitionsfragen kommt, gelegentlich ist ein Ankauf von privatem Land durch die öffentliche Hand nachzuweisen, und sichtbar vollzieht sich die Konfiskationspolitik einzelner Kaiser nach einem klaren, inneren Entwicklungsschema, dessen Tendenz nicht zuletzt den Abbau, zumindest eine verbesserte Kontrolle von Kräften 
und Möglichkeiten bedeutet, deren Gefährlichkeit die Folge ihres wirtschaftlichen Potentials sein könnte. Bei Konfiskation muß Pächtern die bereits bezahlte Pacht zurückerstattet werden, und für die Streichung der reliqua colonorum, der Pachtrückstände, sind die Hinweise in Digesten wie etwa anderen Quellen so, daß Verpachtung gelegentlich fast schon als eine Belastung für Grundeigentümer erscheint.

Wohl sucht man das Fortbestehen dieser Schicht zu sichern, die Scheidung in honestiores und humiliores mit ihren straf- wie zivilrechtlichen Konsequenzen ist die bewußte, nicht ohne Grund durchgehaltene Zementierung sozialer Verhältnisse und Unterschiede, zugleich auch eine Ausweitung unter anderen Vorzeichen. In der Besitzbehandlung jedoch zeigt sich das Bemühen, Durchlässigkeit zu wahren und den Zugang für die unteren Schichten nach oben offenzuhalten. Die Möglichkeiten sozialer Mobilität in der Tat waren vielfältig, auch wenn in diese Zwischenstufen einkalkuliert werden mußten. Es ist aber die Möglichkeit einer solchen Unterwanderung, die jener Verbindung der Bereiche im Sozialen, Wirtschaftlichen und schließlich im Politischen ihre Flexibilität verleiht. Das Interesse für diese unteren Schichten aber dominiert unverkennbar. Es ist ein ebenso brillanter wie komischer Gedanke, einen scheinbar offenkundigen Rückschritt von Zivilisation in der Kaiserzeit darauf zurückzuführen, die Abhängigen seien am Fortschritt uninteressiert gewesen und hätten komplizierte Arbeitsgeräte abgelehnt oder böswillig ruiniert. $\mathrm{Zu}$ belegen ist dies nicht. Belegt werden aber können, um es noch einmal zu wiederholen, die offiziell geförderten Vorteile gerade dieser unteren Schichten, die Möglichkeit von Vermögensbildung zu persönlicher Verfügung durch Eigeninitiative und damit ein Anteil auch an der Produktion. All dies aber muß wahrgenommen worden sein. Der Begriff Fortschritt zwar scheint in letzter Zeit nachgerade zur Phrase geworden zu sein, und die Subjektivität der Auslegung hat in der zweiten Hälfte des vergangenen Jahrhunderts sich zu wahren Orgien aufgeschwungen. Aber wenn es ein Beispiel für solchen gibt, auf überschaubarem Raume verwirklicht, dann ist es die römische Kaiserzeit in den hier angesprochenen Bereichen. Kein Wunder, wenn Zeugnisse gerade dieser unteren Schichten neben gelegentlichen Klagen auch von jenen Aufstiegsmöglichkeiten sprechen und - anders als etwa im Bereich antiker Bürger- und Polistugend - privater Fleiß und Sparsamkeit ihre Belohnung finden. Unseren Kenntnissen nach muß dies für Freie wir für Sklaven zutreffen. Und es fragt sich, wie weit bzw. wie lange angesichts der allgemeinen Bedingungen der Stand des letzteren noch als pudendum empfunden worden sein kann, dessen Aufhebung die Mühe lohnte.

$$
\text { * }
$$

Dies wäre eigentlich Vorbemerkung. Daß hier nur Kriterien angedeutet werden können, manches aber unter den Tisch fallen muß, ist klar. Sicher, es müßte wohl von Fall zu Fall der zeitliche Hintergrund mehr beleuchtet werden, und ähnlich von Fall zu Fall auch ein regionaler Zusammenhang, der Variationen zeitigen mag. Gleiches gilt für eine natürliche Fernwirkung einzelner Gesetze, die sich nur vermuten läßt. Die Prinzipien aber bleiben die gleichen. Die Ernährung jedenfalls unter solchen Voraussetzungen scheint für deren erstes Jahrhundert gesichert, und darauf kommt es an. Gesichert ist denn auch die allgemeine Stabilität des wirtschaftlichen Gefüges als die Grundlage aller Wohlfahrt, die Zeugnisse staatlicher, communaler und privater Prosperität sind in der Tat in keinem Imperiumsteil zu übersehen. Anderes dagegen scheint 
von geringerem Belang. Erkennen läßt sich dies nicht zuletzt daraus, als in der nun folgenden Zeit der angedeutete Zustand sich zu verändern scheint. Für das 3. und 4. Jhdt. jedenfalls sind die im 2. und 3. geltenden Tendenzen so gut wie außer Kraft, ja wird trotz Christianisierung und constantinischer Wende eine Brutalisierung der Verfahrensweisen gerade in diesen Dingen unverkennbar. Den theokratischen Intentionen Constantins braucht derartiges nicht zu widersprechen. Eher im Gegenteil, geht man von deren pragmatischen Hintergründen aus. Sicher - äußerlich mag ein Übergang vorhanden sein.

So gehen die Digesten inhaltlich scheinbar nahtlos in die Gesetzescorpora des Theodosianus und des Justinianus über: Ihre Betonung durch den letzteren bedeutet ein Programm, das auch in der Zeit nach ihm nicht ohne gewisse Fortwirkung blieb, mochte einem Justinian später auch daran gelegen sein, durch die christliche Ethik bestimmt, die härtesten der überkommenen Bestimmungen zu mildern. Und die früher angedeuteten Denk- wie Deutungsweisen bleiben die ganze Völkerwanderungszeit hindurch in Kraft, die Gesetzgeber berufen sich oft genug auf die Tradition. Bestimmt aber scheint der historische Hintergrund von der Caesur, die das 3. Jhdt. bedeutete, und von den Zwängen, die unvermeidlich waren. Denn Gewaltanstrengungen waren seit der Severerdynastie nötig, etwas wie die angedeutete innere Konsistenz aufrechtzuhalten und die sich nun nicht mehr lediglich abzeichnende, sondern physisch hereinbrechende Katastrophe zu verhindern. Von der Rhein- bis zur Donaumündung hält ein halbes Jahrhundert eine Invasionslawine an und macht den Norden des Imperiums weitgehend zur Wüste: Er fällt als Versorgungs- und Steuerträger aus. Im Osten bedeutet das Sassanidenreich einen Neuanfang in den großen Auseinandersetzungen seit Augustus und zwingt verstärkt neue Belastungen auf. Prüft man die Zeugnisse in ihrer Fülle und Vielfalt, man wundert sich, daß trotz allem der Reichsapparat noch funktionierte, ein Fortleben unter überkommenen Bedingungen möglich war und der allgemeine Zusammenbruch sich überhaupt aufhalten ließ. Unterschleife, Bestechlichkeit und die entsprechenden Verhaltensweisen der Betroffenen von beiden Seiten sind die natürliche Folge im kleinen, sie erklären sich neben einer gewissen Tradition aus dem Zwang zum Überleben als entsprechende Variationsform. Im großen kommt man um die gewaltsame Anpassung scheinbar unverrückbarer staatsethischer Prämissen an die neue Lage nicht herum, selbst Entwicklung und Ausprägung einer gleichsam lückenlosen Bürokratie gehören dazu. Jammer über diese Entwicklung findet sich in Fülle - aber in gewisser Hinsicht war es vielleicht sogar die ganz natürliche neue Interessengemeinschaft aller Beteiligten, die den endgültigen Zusammenbruch mit verhinderte, nachdem sie sich gleichsam natürlich herausgebildet und ihre Glieder, Kaiser, Verwaltung, soziale Schichtung und Armee, in ein bisher kaum denkbares Verhältnis gegenseitiger Abhängigkeit voneinander gebracht hatte.

Was die erhaltenen Gesetzescorpora erkennen lassen, sind Versuche, diese Abhängigkeit zu festigen. Aber es ist zugleich zu erkennen, wie schwer dies war. $\mathrm{Zu}$ den überhöhten Kosten einer nunmehr geradezu verzweifelten Foederatenpolitik angesichts wachsender Bedrohung kommen die für eine Armee, die unter Diokletian das Fünffache von der unter Augustus ausmachte: Ihr Barbarencharakter bedeutete eine Verringerung der Kosten kaum. Man mag von den notwendig gewordenen Hilfeleistungen für die barbarischen Verbündeten absehen, die an Umfang wuchsen und sicher dennoch an keiner Stelle ausreichten. Für das Imperium vervielfachen sich damit die Ausgaben bei geschwundenen oder immer mehr sich verkleinernden Einnahmequellen, von geregelter und einer immer noch zentral zu steuernden Versorgung der ge- 
samten Bevölkerung kann aus vielen Gründen kaum mehr die Rede sein. Daß die Wirtschaft unter solchen Bedingungen für alle Beteiligten zu einer Zwangswirtschaft werden mußte und auch, daß oben angedeutete soziale Entwicklungstendenzen abbrechen, ist vor solchem Hintergrund das geradezu Natürliche, und gleiches gilt für einen unvermeidlichen, ganz natürlichen Prozeß von Regionalisierung in allem, was zum Leben gehörte, weil anders eine Sicherung der allgemeinen Grundlagen nicht möglich war. So reglementiert man in den Städten mittels der Kollegien die Produktion, d. h. das gesamte Erwerbsleben selbst auf kleinstem Raume, und bringt sie dadurch völlig unter eine Kontrolle, die das Staatsmonopol in verschiedenen Variationen zur Funktion von etwas macht, das an den totalen Krieg des 20. Jahrhunderts, hier freilich in Permanenz, erinnert. Auf dem Lande hingegen scheint das Bemühen, einem deutlichen, mit der Zeit noch wachsenden Menschenmangel durch Verwendung williger Barbaren zu begegnen, eine erneute Gefahr, fehlt jetzt doch weitgehend eine integrierende, zumindest assimilierende Bewohnerschicht, und dies wenngleich nicht überall, so doch gerade in den Landstrichen, auf die es ankam. Die Gesetzestexte jedenfalls lassen das Bemühen um Gewinnung von Arbeitskraft nicht übersehen - um die in erster Linie ist es bei allem gegangen. Die andere Seite entspricht dem: Streiks, Arbeiterflucht etc. hatte es in der Antike seit der hellenistischen Zeit immer wieder gegeben.

Was wir nunmehr sehen, ist der Versuch ganzer Bevölkerungsgruppen, sich ihren Verpflichtungen und auch ihrem Status zu entziehen, nicht weil es an Arbeit mangelt, sondern weil angesichts der Belastung alles an Fleiß und Mühe nicht mehr ausreichten, das Leben zu fristen. Alternativen freilich waren selten. Versorgungsschwierigkeiten nehmen zu oder lassen sich kaum mehr kontrollieren, es sei denn, wie angedeutet, auf kleinstem Raume, doch die staatlichen Koerzitionsmaßnahmen reichen an keiner Stelle mehr aus. Daß es bei all dem mit sozialer Caritas allein nicht getan war, um der Not abzuhelfen, die direkt oder zumindest indirekt fast die ganze Imperiumsbevölkerung betraf und deren Folgen ein undurchdringliches Geflecht von Kausalitäten bildeten, darüber war man sich im klaren. Nein, was hier half, waren allein drastische Maßnahmen, war Rigorosität, und dies im Guten wie Bösen. So feiert die HA unter Alexander Severus $(21,2)$ ein Programm, das den Ankauf fruchtbaren Landes vorsah, um dies an Ärmere zu verpachten und dabei die Pacht in Naturalien einzutreiben: Die Nachricht scheint weniger wichtig wegen aufgezeigter Möglichkeiten als deshalb, weil die gleiche Maßnahme auch aus dem Jahr 405 als Gesetzestext überliefert ist. Nicht daß man beabsichtigte, den Großgrundbesitz zu zerschlagen, im Gegenteil, denn dessen Funktion wurde jetzt noch einmal eine andere, wichtigere. Die Phänomene, wie angedeutet, sind aus den Quellen bekannt oder ergeben sich aus der Erwägung als Folgerung. Vor einer Generalisierung aber wie vor einer Simplifikation warnen alle ernst zu nehmenden Arbeiten zur Sache. Zu viel, trotz aller Deutungsversuche, ist unbekannt, und weder allgemeinverbindliche Termini noch unsere Kenntnis regionaler Gegebenheiten helfen weiter.

Was sich zwangsläufig aber zu ergeben scheint, ist eine Akkumulation von Land in den Händen einzelner bekannter Familien, die zu Streubesitz im ganzen Imperium führt, und notgedrungen zur Einführung privater Institutionen, die diesen Besitztümern den Charakter kleiner, in sich selbst geschlossener Staatswesen gibt. Die Stelle indes zeigt, wie man jetzt zwischen den Faktoren zu lavieren hatte, auch wenn eine entsprechende Entwicklung geraume Zeit bereits sich abgezeichnet hatte. An sich hat die Umstellung zu einer Produktion in großen Einheiten, sachlich und von außen her gesehen, 
viel Verwandtes etwa mit der modernen Wirtschaftspolitik ohne Unterschied zwischen einer kapitalistischen oder einer sozialistischen Zielsetzung und entspricht denn auch bis zu einem gewissen Grade den Erwägungen zu einer Effektivität durch Konzentration der Möglichkeiten und zur Bildung immer größerer Komplexe. Bedeuten, wie angedeutet, die Collegia im Gewerbeleben von vornherein eine Tendenz zur indirekten Verstaatlichung in fast allen, besonders den wichtigen und überhaupt einträglichen Gebieten, auf dem flachen Lande wird das Entstehen der Großvilla wie umfangreicherer Einheiten besonders zur Produktion von Nahrungsmitteln darüber hinaus offensichtlich immer stärker gefördert oder aber lediglich damit forciert, was stets vorhanden war. Der Großbesitz bildet, privat oder öffentlich, eine Wirtschaftseinheit, in der die verfügbaren, an Zahl meist zu geringen Kräfte spezialisiert und damit am effektivsten verwendet werden können. Daß in solchen Zusammenhang Rechtskategorien dieser Beschäftigten an sich noch weniger eine Rolle spielten als zuvor, liegt auf der Hand, die öfter definierte Funktion, die Wahrung des Steueraufkommens und die Aufrechterhaltung der Ordnung bis hin zu lokalgerichtlichen Konsequenzen macht diesen Großbesitz erst jetzt zu einer Institution staatlichen oder halbstaatlichen Charakters. Wohl hatte bereits Varro Ähnliches vorgeschlagen. Indes, sein Ziel war alles in allem noch ein gewisser Profit gewesen. Hier geht es allein um die Erfüllung offizieller fiskalischer oder politischer Verpflichtungen, um das Verhältnis von Erwirtschaftetem und von Steuer, d. h. um das allgemeine Überleben schlechthin.

Hand in Hand damit aber verläuft, das ist nicht zu leugnen, wiederum ein Prozeß der Zerschlagung und Auflösung von Privatbesitz und Privatwirtschaft in kleinem Rahmen, d. h. von Entwicklung zum Zwangscolonat und damit vom Ende der wirtschaftlichen Selbständigkeit für einen großen Teil der Reichsbevölkerung, der sich in Abhängigkeit solventer Grundherren begeben muß, und alles in allem die erweiterte, institutionalisierte Fortentwicklung der Patronatsverhältnisse der Republik, jetzt unter anderen Vorzeichen, aber dringender als je zuvor. Es ist dies kein Prozeß von Gewalt und Unterordnung, sondern eher von Anpassung an Umstände, die sich anders nicht bewältigen lassen. Daß unsere Überlieferung, Gesetze wie literarische Zeugnisse, nur Fehlentwicklung und negative Aspekte hervorheben, ist nicht zu verwundern. Im wesentlichen aber hat die Institution ihren $Z_{w e c k}$, die allgemeine Stabilisierung, erfüllt, so gut dies möglich war. Sicher, das Imperium in der Vielfalt seiner Aufgaben und Belastungen kam ohne eine ausgeprägte, sich verzweigende Bürokratie nicht aus. Patron wiederum, Patrocinium und alles, was damit in einem Zusammenhang steht, regional oder überregional wirksam, ist ein zeitloses Phänomen: In der Spätantike bedeutet es zu dieser Bürokratie ein Korrektiv, das sie aber dennoch unterstützt und sie erst effektiv macht, indem es ihr einiges an Arbeit abnimmt, bei aller Selbständigkeit aber ebenso der Kontrolle unterworfen und selbst in das Steuer- wie Finanzgefüge der Verwaltung eingegliedert, indes anderseits in der Lage, Konflikte zu verhindern, an denen die Verwaltung scheitern muß. Die Ambivalenz in der Administration ergänzt in ihrer Weise damit die innerhalb der sozialen Struktur, und es bleibt zu fragen, wie weit wir selbst uns gerade hier durch die mehrfach angedeutete Verwischung sachlicher und juristischer Termini täuschen lassen müssen. Zeugnisse dafür, daß eine solche Gleichsetzung in der Bezeichnung als eine Schande oder zumindest als lästig empfunden wurde, gibt es nicht.

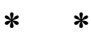

$*$ 
In den Städten wird die Decurionenschicht bei der Wahrnehmung ihrer Steueraufgaben zwangsläufig so gut wie ruiniert, dies freilich gegen die Absicht aller Beteiligten oder Betroffenen, auf dem Lande ist der Colone fest in der Steuergesetzgebung verankert und seine Arbeitskraft wird zur Verrechnungseinheit, die sich weder umwandeln noch veräußerlichen läßt. Der Erbzwang des Colonats aber bedeutet den notgedrungen geförderten Verfall aller früheren Pacht- und Mietvorstellungen in diesem Bereich. Ihn ebenfalls lediglich als Verschlimmerung zu sehen, aber wird den Dingen nicht gerecht. Man pflegt zu vermuten, es sei dies der erste Schritt zu einer unaufhaltsamen Zwangsversklavung ländlicher Bevölkerung und zugleich damit das erste Symptom einer nun um sich greifenden Feudalisierung, was immer der Begriff bedeuten und beinhalten mag. Man vergißt dabei freilich, daß der Erbzwang auch für alle anderen Berufe gilt, und natürlich für das Militär. Jedoch, indem man den Menschen an den Boden als seine Rolle im gesellschaftlichen Gefüge fesselt, scheint eine Garantie gegeben, daß dieser Boden genutzt wird und weiter Nutzen bringt, daß Steuereinkünfte gesichert bleiben und so zugleich das allgemeine Gefüge gehalten wird. So wird der an sich freie Pächter zum Inventar, der Typ des adscripticius oder glebae adscriptus zum Charakteristicum des 4. Jhdts., es fehlt nicht an drastischen Verboten gegen unerlaubtes Verlassen von Stand und Arbeitsplatz für die an sich rechtlich freien, selbständigen Menschen. Die Tatsache einer Gültigkeit entsprechender Verpflichtungen selbst bei geleisteten Abgaben und ordnungsgemäßem Verhalten indes ist dabei ebenso bedeutsam wie die, daß ein Colone selbst innerhalb einer Wirtschaftseinheit nur mit Einschränkungen etwa von einer zur anderen Stelle versetzt werden darf. Die Frage nach dem Schicksal der Colonen bei Güterverkauf scheint bis in die byzantinische Zeit strittig geblieben zu sein. Da, wo es dennoch zur Flucht kam, mag sich diese zum großen Teil mit aus dem Mangel an Arbeitskräften und den deshalb stets vorhandenen besseren Bedingungen für Einzelne mit erklären. Aber eine allgemeine und permanente Fluktuation ist zu bezweifeln.

Daß mit diesem Colonen nun der Sklave fast ausschließlich in einem Zuge genannt wird, verwundert kaum, ihre Funktionen, vom Ganzen aus gesehen, unterschieden sich auch so gut wie nicht voneinander. Immerhin, dieser Sklave war leichter an den Boden zu binden. Gelegentlich bezeichnet man sogar seine Arbeit geradezu als munus sordidum, was eine vielleicht sogar provozierte weitere Begriffsverwirrung schafft oder aber wirklich vielleicht auch spiegelt: Gelegentliche Betonung der Rechtsunfähigkeit scheint auf den ersten Blick nunmehr übertriebene Reaktion auf die angedeutete frühere Veränderung der äußerlichen Bedingungen und eine allzu weit bereits entwikkelte Selbständigkeit dieser Gruppe. Offensichtlich verlieren im 4. Jhdt. die humanisierenden Tendenzen in der Tat denn unverkennbar wieder an Kraft, auch wenn sich an der Situation wenig ändert. Die Möglichkeiten von einer Anklage der Herren durch Sklaven werden eingeschränkt, man stigmatisiert jetzt Flüchtlinge, wobei freilich zu erwähnen wäre, daß dies im Militärdienst lange schon geschah. Seit 368 gibt es eine Gesetzgebung gegen Herumtreiber, anderseits wird strengstens auch die Rückführung kriegsgefangener Sklaven gefordert, aus dem Militärdienst sind sie ebenfalls zurückzuholen und ihrer zivilen oder privaten Verwendung wieder zuzuführen, selbst wenn sie bereits Rang und Auszeichnungen erlangt haben sollten. Die Frage freilich, wie oft dies wirklich geschah, zwar wird kaum zu beantworten sein, die Militärbehörden hatten sicher Möglichkeiten genug, entsprechende Anforderungen zu ignorieren. 
Dies sind Einzelbeispiele, ein Gesamtbild wohl müßte diffiziler ausfallen. Der Hintergrund dieser Verhärtung allerdings scheint durchsichtig, gelegentlich wird er sogar formuliert: Es ist einmal schlechthin die Erhaltung der Arbeitskraft unter Bedingungen, die schwieriger geworden sein müssen, und es ist anderseits das Bemühen, die allgemeine Katastrophe und eine durch die anhaltende, ausweglose Ausnahmesituation ins Gleiten gekommene Sozialstruktur nicht noch weiter durcheinander zu bringen, und mit ihr denn zugleich auch die wirtschaftliche. So wird es auch für den Sklaven von nun ab wohl schwerer, sich seiner Lage zu entziehen, oder auf seine Befreiung hinzuarbeiten, Constantin gesteht dem Besitzer wieder Straffreiheit zu, wenn die Tötung eines Sklaven nicht im Zorne, sondern zur Bestrafung geschieht. Er betont damit offenkundig, was außer Gebrauch gekommen war, doch auch hier schienen Zweifel an einer Durchführung angebracht. Zwar scheint es im Erbrecht gewisse Möglichkeiten für Kinder aus Mischehen zwischen Sklaven und Freien zu geben, die Gesetzestexte lassen auf eine nach wie vor praktizierte Freilassung schließen, die Heirat zwischen Sklaven und Freien aber ist nun offensichtlich verpönt, Constantin scheint gerade hier eine durchlässige Grenze im Rahmen seiner Reorganisationsbemühungen versperrt zu haben, trotz Christentum und dem Postulat der Milde in einer ganz neuen Sicht hier auffallend reaktionär.

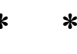

So mag sich die Frage nach der Wechselbeziehung von Religion und Politik zueinander auch von hier aus stellen. Daß Constantin die Synthese noch nicht gelang, ja gar nicht gelingen konnte, lassen die einschlägigen Gesetze der eigenen wie mehr noch vielleicht späteren Zeit erkennen, auch wenn diesen deutlich eine Absicht des Modifizierens, Anpassens und alles in allem des Ausgleichs immanent ist, der seinen Zielen dennoch am nächsten gekommen sein wird.

Äußerlich gesehen wohl dienen die erwähnten Bestimmungen nicht zum wenigsten einer erneuten planvollen Stärkung von Oberschicht, von Grundherren, Großpächtern und, wenn man so will, von Magnaten, noch im Rahmen der Reformen, mit denen Diokletian begonnen hatte, weil man ihnen gerade jetzt eine Garantie wirtschaftlicher und sozialer Stabilität zutrauen konnte, so daß ihre Förderung, Erleichterung und Heraushebung berechtigt war. Bei näherem Zusehen indes zeigt sich, daß dieser Gedanke auch jetzt täuscht. Denn trotz einer Vielfalt an scheinbaren Privilegien aus Immunitäten, trotz Heraushebung als honestiores, die Gesetzgebung schränkt auch sie ein, so daß dieser Magnat, solange sie wirksam blieb, an seine Stelle nach wie vor nicht weniger gefesselt war. Sicher, ohne seine Genehmigung kann weder ein Sklave noch ein Colone eine Transaktion vornehmen: Es gibt indes eine Fülle von Verordnungen besonders im Erb- und Familienrecht, die direkt oder indirekt betonen, daß auch seine Funktionen in dem politisch-gesellschaftlichen Gefüge bei aller scheinbaren Überhöhung und trotz äußerlichen Machtzuwachses angesichts erwähnter Funktionen der allgemeinen Stabilisierung dienten und allein vom Fiskalismus bestimmt waren. Offizieller Regelung für diesen Besitzer unterliegen z. B. nicht nur die Fragen der Güterteilung, sondern selbst die von Verlobungszeit und Mündigkeit.

Hinter dieser Relativierung aber steht die offizielle Furcht vor Auflösung und Zerschlagung bestehender Wirtschaftseinheiten, zumindest vor der Schmälerung ihrer Effektivität. Sie diktiert denn wohl auch die Verbote allzu 
eifrigen Ausgreifens einzelner an sich interessierter Personen oder des Sichverzettelns etwa in künstlichen, fragwürdigen Patronaten. So mag das sogenannte patrocinium vicorum Bereicherung und Machtzuwachs im kleinen bedeuten: Im Krisenfall verband sich mit ihm eine besonders gravierende Belastung und notfalls die Verringerung der eigenen Substanz, was überdies zu einer Konkurrenz mit den legal fungierenden Grundbesitzern und damit einer Verwirrung bestehender wirtschaftlicher und sozialer Verhältnisse führen mußte. Das Interesse von Betroffenen an solcher Konkurrenz, Colonen, Sklaven, bedeutet eine Rechtfertigung für diese Rolle nicht.

Doch sind die einschlägigen Gesetzestexte ohne Schroffheit, man ist versucht, zu folgern, das patrocinium sei eine Zeitlang geduldet worden als zumindest provisorische Stütze, sei es dort, wo der eigentliche patronus mit seinen Möglichkeiten am Ende und nicht mehr in der Lage war, seinen Aufgaben nachzukommen, oder aber, wo die finanzielle Bedrückung der Abhängigen zwar zur unerträglichen Belastung geworden war, ein rigoroses Eingreifen aber dennoch andere Schwierigkeiten heraufbeschworen hatte. Es wird denn einmal sogar als dementia etwa für erfolg- und vermögensreiche Personen bezeichnet, mit Sondersteuern belegt und 415 ganz abgeschafft. Das, was Salvian über dieses patrocinium für die Folgezeit in Gallien schreibt, mag den dortigen Verhältnissen entsprechen, die zweifellos eigener Art waren und im 5. Jhdt. an Härte zunahmen, wobei von einer staatlichen Kontrolle kaum mehr die Rede sein kann.

Doch es bleibt zu fragen, ob es überhaupt eine Beziehung zu dem gibt, was die einschlägigen Gesetzestexte umschreiben. Im übrigen, wir wissen nicht, wie wir die Kategorie der Grundbesitzer nach oben wie nach unten abzugrenzen haben, welche Möglichkeiten und Wege es gab, zu reüssieren und die gewonnene Basis weiter auszubauen. Ihre Zahl, auch die der illegalen, war sicher groß. So bleibt uns auch verborgen, wie deren Anteil an der Gesamtzahl einzuschätzen ist, die zu dieser Gruppe gehören, und wie viele im Laufe der Jahrhunderte gescheitert sind. Unsere Zeugnisse vom Standes- und Elitebewußtsein der Erfolgreichen lassen vermuten, daß dies viele waren. All dies gilt denn für den Decurionenstand im besonderen. Regionale Unterschiede wie bei allem, was hier angedeutet ist, gab es, sie beeinflussen indes die Probleme auch jetzt nicht. Grundbesitzer auch er, aber ohne die Vorteile der hohen Aristokratie bestand seine Rolle, ohne klare soziale oder rechtliche Abgrenzung nach oben wie nach unten, doch ohne wirksame Privilegien, innerhalb der Civitas als eigentlicher Steuerträger neuerdings vorwiegend darin, Steuergelder vorzustrecken und so die Wirksamkeit des Systems zu gewährleisten, zugleich aber den Druck auf untere Schichten abzumildern und die völlige steuerpolitische Stagnation so gut es ging zu verhindern. Es ist hier ohne Belang, wie weit entsprechende Analogien im hellenistischen Ägypten oder etwa griechische Liturgievorstellungen eine Rolle bei Genese und Entwicklung des einschlägigen Aufgabenkreises eine Ursache sind, über bloße Anregungen hinaus hatte das Imperium seine eigenen Probleme, die entsprechende Institutionen verlangten. Eine für das gesamte Imperium gültige, einheitliche Berechnungsgrundlage der Vermögen für die Zugehörigkeit zwar gab es nie, aber daß durch die allgemeine Belastung im 3. und noch im 4. Jhdt. der Stand zum Ruin gelangte, war unausweichlich, hatte er doch wohl selten nur die Aussicht, Außenstände solcher Art ohne weiteres wieder eintreiben zu können. Der lokale Euergetismus kam hinzu, seinen Forderungen sich zu entziehen, war unmöglich. Doch das Schwinden von Zeugnissen in der späten Zeit, läßt erkennen, daß es eine breite Schicht nicht mehr für eine solche Rolle gab, die 
Aufhebung der Funktionen unter Justinian ist Vereinfachung und Erleichterung zugleich. Entsprechendes zu leisten vermochten nur noch die allgemein bekannten schwerreichen römischen Familien, und von hier zur caritas etwa der begüterten Damen der Gesellschaft ist kein großer Schritt.

Der Kompliziertheit von Stand und Rolle entsprechen wohl Möglichkeiten, durch Eintritt in Militär, Verwaltung und Kirche sich diesem Status zu entziehen, nach CTh 12, 50, 1 müßte auch wohl die Übernahme kirchlicher Verpflichtungen eine gewisse Erleichterung gebracht haben. Ähnlich wie den Sklaven oder den Colonen aber holt man auch seine Mitglieder von dort zurück, und es fragt sich, was dagegen von Fall zu Fall, anders wohl als bei der Armee, der Einspruch der betreffenden Institutionen vermochte. Die Wiederholung einzelner Bestimmungen in den Gesetzescorpora freilich läßt eine stetige Unterwanderung vermuten, Erleichterungen in solchem Zusammenhang sind nicht zuletzt von der Frage eines personalen Ausgleichs her zu verstehen. Kein Wunder, daß die Zahl der Verordnungen über eine Rückführung oder über den Erbzwang etc. die bezüglich der Colonen und Sklaven noch übertrifft. Gelegentliche Versuche der Erleichterung der Lage für diese Decurionen scheinen hier wenig zu besagen gegenüber der Kontrolle von Besitzveränderung und etwa von Pachtverbot außerhalb der eigenen Munizipien, oder aber den Strafen für die Vergehen von Manipulation in Steuerfragen. Bei all dem hat der Gesetzgeber immer wieder zu klagen, derartige Mitglieder der Oberschicht entzögen sich ihren Pflichten durch eine Flucht aufs Land, begäben sich als Colonen in Abhängigkeit, ja heirateten sogar Sklavinnen und lieBen sich trotz Strafe von Verbannung und Versetzung der Nachkommen in den Sklavenstand nicht davon abbringen. Sie zeigen, was hier möglich war, zugleich freilich auch, welchen Gefahren innerer Auflösung in der Tat hier begegnet werden mußte.

Alles in allem, es ist auf den ersten Blick das Bild eines Zwangsstaates, bestrebt und wohl auch ohne wirkliche Alternative, um stabiler Verhältnisse willen das Letzte von seinen Untertanen zu fordern. Rücksicht und Wohlfahrt werden in Gesetzestexten gelegentlich betont, sind aber faktisch unmöglich zu realisieren. Aber das Bemühen, in einer aussichtslosen finanziellen Lage zu tun, was sich tun ließ, für alle Seiten das Schlimmste zu verhüten, das ist dennoch unverkennbar. Und die Rolle von Decurionat oder Colonat stehen demnach in einer deutlichen Analogie zueinander. Es ist hier wenig von Belang, daß im Osten offensichtlich die Dinge sich nach der Trennung von 395 bald wieder stabilisierten, ja in der 2. Hälfte des 5. Jhdts. die Staatswirtschaft aus Steuergeldern gelegentlich sogar einen beträchtlichen Überschuß herauswirtschaften konnte.

Die vorläufigen Folgen sind, sozial gesehen, ein Auseinanderklaffen des sozialen Gefüges und wohl die Zerstörung mittlerer Schichten, die in der hohen Kaiserzeit immerhin noch zu vielfältigen Zeugnissen ihres Selbstbewußtseins in der Lage gewesen waren, sind ein Zunehmen der Zahl Armer, Hoffnungsloser, Depossedierter und ohne eigene Schuld wurzellos Gewordener, und dies am meisten in den Provinzen, die vielfältige Last zu tragen haben. Sie manifestieren sich in organisierter Form in der Circumcellionenbewegung in Afrika und in der der Bagauden in Gallien wie in Spanien, wie auch darin, daß diese Bewegungen auf verschiedene Bereiche menschlichen Lebens übergreifen und trotz staatlicher Bemühungen entweder um Ausgleich oder um Zerschlagung sich nie ganz unterdrücken ließen.

Nicht übersehen freilich darf in diesem Zusammenhang die Rolle der Kirche werden, und von deren Möglichkeiten wird man nicht zuletzt mit aus- 
gegangen sein, als ein Constantin, pragmatisch genug, die Wende vollzog. Sicher, sie wird schnell selbst Grundbesitzer: Aber als Asyl und in schrankenloser Nutzung ihrer Möglichkeiten tut sie Wesentliches zur Linderung der größten Not. Sie ersetzt damit, und zwar ganz anders als dies je eine noch so breite Oberschicht von Grundbesitzern hätte tun können, den Staat in einer Reihe von Aufgaben, vor denen er um seiner selbst willen die Augen zu verschließen hat. Und ganz sicher erklärt gerade diese Funktion mit die Wende unter Constantin. Daß sie zugleich zur Konkurrenz in öffentlichen wie offiziellen Belangen werden konnte, wußte man zweifellos, nahm es aber nicht ohne Erleichterung in Kauf.

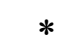

Erklären läßt sich, wie gesagt, diese Anpassung des Staates an seine Rolle als Versuch der Kräftekonzentration in allen Bereichen, als Totalisierung, wenn man so will, die hier sich abzeichnet. Ihre Wurzeln freilich sind früher, lange vor der eigentlichen Katastrophe, zu suchen. Diokletian wie Constantin greifen zweifelsohne auf ein Erfahrungsreservoir zurück. Ich selbst möchte meinen, selbst jene Constitutio Antoniniana 212 angesichts bevorstehender kriegerischer Anstrengungen von ganz besonderen Ausmaßen unter Caracalla müsse in diesem Rahmen gesehen werden. Auffallend auch, daß Verordnungen, Gesetze und Strafandrohungen in den überlieferten einschlägigen Sammlungen sich ständig wiederholten, Beweis nicht nur dafür, daß man sie von allen Seiten her immer wieder zu umgehen suchte, und demnach auch dafür, wie der Druck in der Tat auf allen, den Magnaten wie dem Kleinen, Ärmsten gelastet haben muß. Habgier und sinnloser Lebensgenuß, allgemeine Interesselosigkeit bzw. das Bemühen, aus den Dingen das beste für sich zu machen und dabei zimperlich weder gegenüber der Allgemeinheit noch gegen Einzelne zu sein, wird auch von den literarischen Quellen als Zeitsymptom geschildert oder ist ein ungewolltes Ergebnis von Selbstdarstellung: Vor dem Hintergrund offizieller Bemühungen, zu halten, was zu halten ist, erwecken sie den Eindruck einer Gesellschaft, die fatalistisch der unausbleiblichen Katastrophe gegenüber steht und gelegentlich noch zum Tanz auf dem Vulkan ansetzt. Die Flucht in eine sublimierte Welt von geistiger Tradition ist die andere Seite und ein natürliches Pendant. Sehr echt freilich wirken die entsprechenden Äußerungen und Bekenntnisse der bekannten Persönlichkeiten auch nicht.

Rostovtzeff spricht vom Kampf zwischen Stadt und Land als einem bestimmenden Kriterium dieser Zeit, Marx und Lenin erkennen die natürlichen Geburtswehen des Feudalismus. Stalins Sprachregelung kombiniert aus den Zeugnissen einen Klassenkampf, was sowjetische Historiker seither wieder revidieren und zu mildern suchten, die westliche Historiographie laviert zwischen den Fronten, und erschöpfend Neues hat sich an Erkenntnissen seither nicht ergeben. Nur darüber ist man sich einig, daß zur äußeren die innere Katastrophe tritt. Und ganz unter Kontrolle bringen ließen sich Protestbewegungen bis an das Ende der Antike nicht, unter welchen Vorzeichen sie auch begonnen hatten. Wie gesagt, der Unausweichlichkeit der eigenen Lage scheint man sich unter den Zeitgenossen ohne Unterschied bewußt.

Erleichterungen in kleinem Maße führt, freilich ohne Erfolg, fast jeder Kaiser noch durch, indes, Zeugnisse auch von Anklage gegen Kaiser und Staat fehlen nicht, ja die Zahl gegenteiliger Bekenntnisse nimmt gegenüber früheren Jahrhunderten zu. Wir wissen aber anderseits von keinem der Usur- 
patoren des 4. oder 5. Jhdts., daß er sich etwa durch Versprechen wirtschaftlicher Erleichterungen Zustimmung erkauft hätte. Auch finden wir nicht, daß man sich, was nahe gelegen hätte, allgemein durch eine Flucht über die Grenzen oder Verrat wirklich allgemein die Rettung erhofft hätte, so wie dies ein Salvian darstellt, auch wenn anderseits eine vielfältig sichtbare nuancierte Verfremdung aller Schichten sichtbar wird, jede in anderem Bezug.

Sicher, es gibt vereinzelte Zeugnisse von Personen, denen es bei Persern, Germanen oder Hunnen als Spezialisten gut ging, sie dürfen indes nicht verführen. Daß unterdrückte oder als solche sich fühlende Gruppen zu einem drohenden Feind überliefen, ist seit den athenischen Sklaven von Dekeleia ein zeitloses Symptom politischer Gefahr, sagt aber wenig für die Situation im einzelnen aus. Und gelegentlich auch wird die Reue über das eigne Verhalten drastisch geschildert. Es bleibt in der Tat zu fragen, wo man Besseres hätte finden können: Die germanische Herrschaft bedeutet fast überall noch die Verschärfung des wirtschaftlichen Druckes wie wohl die der sozialen Verhärtung angesichts anderer Lebensbedingungen, dies möglicherweise aus Hilflosigkeit oder einer Fehldeutung der Verhältnisse durch die neuen Herren im Imperium heraus. Und dies geschieht fast stets an Stellen, an denen sich Rom immerhin noch bemüht hatte, Schlimmstes zu verhüten.

Gängige Deutungen zeichnen das Gesamtbild dieser Zeit denn in dunkelsten Farben. So, wie wir es sehen müssen, freilich wird man ihrer damit aber doch nicht ganz gerecht. Die Voraussetzungen der Imperiumskatastrophe zwar sind scheinbar bekannt. Sie in ihrer Verwobenheit zu durchdringen oder zu differenzieren indes hat noch jeder Bemühung gespottet, mögen Teilaspekte, jeder für sich, auch ihren Sinn haben und auch nicht ohne Kausalität geblieben sein. Die verzweifelten Bemühungen, wie sie sich aus Gesetzestexten und anderen Nachrichten für das Kaisertum entnehmen lassen, seinen Verpflichtungen entsprechend ein Gleichgewicht der Anforderungen zu erhalten, geben derartigen, scheinbar eindeutigen Zeugnissen doch einen anderen Zusammenhang. So stellen wir ein belastendes Zunehmen von Zivil- wie Militärverwaltung fest.

Indes - die Bürokratisierung in dieser Weise war der einzige Weg, dem Einzelnen ein möglichst hohes Maß an Gerechtigkeit der Behandlung widerfahren zu lassen und so die Gesellschafts- und Wirtschaftsstruktur trotz wachsender Gegensätze von Interessenbereichen in der Verwobenheit ihrer Elemente aufrecht zu halten. Einige Jahrhunderte hindurch ist dies in der Tat gelungen. Das Beispiel moderner, über Gebühr armer Staaten mit einem Übermaß an Uniformierten in vielerlei, auch Pseudofunktionen, legt die Vermutung nahe, man habe denn auch damit zugleich nicht zuletzt eine Art Versorgungspolitik versucht. Ein gleiches gilt für die Trennung von Zivil- und Militärressort als einer Verdoppelung von personellen Verwendungsmöglichkeiten. Die von Diokletian begonnene Steuerreform mittels iugatio und capitatio bedeutete zweifellos eine Last. Indiktion und Steuerturnus indessen ermöglichten nicht nur für den Staat, sondern für die am ehesten betroffenen Untertanen wenigstens ein Minimum von Planung auf einen gewissen Zeitraum und einen Schutz vor allzu großer Willkür von welcher Seite immer. Ein gleiches gilt für das Preisedikt, das freilich bezeichnenderweise offensichtlich nur in Teilen des Reiches und auf jeden Fall nur für kurze Zeit zur Geltung kam.

All das freilich ist vordergründig und wohl auch deshalb nie mehr als ein Provisorium gewesen. Wichtiger scheint das weniger Spektakuläre und nicht zuletzt deshalb auf weite Sicht hin Angelegte. Wir haben oben die collegia erwähnt und ihre unterordnende, lähmende Wirkung auf Produktion und Han- 
del. Das ist aber Deutung ex eventu. Für den Augenblick müssen diese nicht nur den Zusammenhalt der Branchen in mehr als kritischer Situation und neben einer Kontrolle doch selbst wohl die Materialbelieferung in einer staatlichen oder zumindest offiziellen Regie bedeutet haben, dazu etwas wie stets auch eine gewisse Absatzgarantie und damit ein wenigstens minimales Element der Sicherung. Die Landwirtschaft etwa kennt den Ausgleich von Material- und Geldsteuer, die adaeratio. Sie hat eine Analogie in den Pachtbedingungen mit ihren Zahlungsmodalitäten. Allgemein gilt sie als Belastung angesichts eines besonderen Versorgungsbedarfs für Heer und Verwaltung. Indes - muß es für die Landwirtschaft nicht auch eine Erleichterung bedeutet haben, ihre Produkte erst verkaufen zu können, um zum Geld für die Steuern zu kommen, und damit nicht allein den Unbilden einer Inflationswährung ausgesetzt zu sein, die endemisch war?

Dazu kommt etwa auch noch denn die Emphyteuse, jene typisch antike Form freiwilliger Bodenbebauung und der Nutzbarmachung unbebauten Landes. Sie wurde von Hadrian erstmals offensichtlich im großen Stil verwendet, um durch Förderung von Eigeninitiative mittels staatlich geregelter Erbpacht etwas wie neues freies Kleinbauerntum auf eigener Scholle zu schaffen, oder zumindest dessen Entstehung zu fördern. Wohl erhält sie seit spätestens Au-

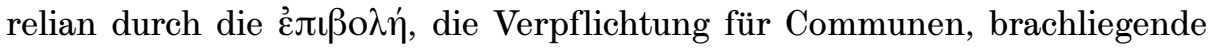
Gebiete mit zu bebauen und sich für sie besteuern zu lassen, wiederum einen gewalttätigen Zug, und dies für den Einzelnen wie für die Communen als Ganzes. Wichtiger aber scheint mir, daß man daneben stets am hadrianischen Aspekt festhielt, d. h. daß Mitglieder der Unterschicht, Colonen und Sklaven, jederzeit Gelegenheit hatten, sich eine steuerbegünstigte wirtschaftliche Eigenexistenz zu schaffen, und so zugleich zwei Lücken zu schließen. Neben gleichmäßiger Verteilung allgemeiner Lasten ist das Festhalten am Prinzip der Eigeninitiative noch während der Völkerwanderung etwas, das zur sonstigen Rigorosität scheinbarer Unterdrückung wirtschaftlich abhängiger Unterschichten in eigenartigem Gegensatz steht und mitten im Zusammenbruch noch die Tendenz jener Stabilisation von innen heraus manifestiert.

Daß die Gesetzestexte gerade in diesem Zusammenhang die Unterschichten gleichsam in einen Topf werfen, fällt auf. Vereinfachung oder saloppe Sprache erscheinen hier wohl als besonders plausibel; eine Disqualifikation braucht für keine der beiden Seiten vorzuliegen. Das gleiche gilt für die Terminologie, die etwa zwischen Personen- und Steuerrecht nie klar unterscheidet. Eindeutig ist kaum einer der Begriffe, um die es geht, aber dies gehört zu der erwähnten Pragmatik von zeitlosem Charakter. Gelegentliche Unterschleife und die Ausnutzung der Steuererleichterungen durch Unbefugte, gegen die einzuschreiten war, stehen dazu nicht in Gegensatz, sondern scheinen all dies noch zu bestätigen.

Dazu kommt indes noch ein anderes. Wohl beinhaltet die Bindung an den Boden, die sich immer mehr intensiviert, für die jeweils Betroffenen eine Form der scheinbaren Verknechtung, die man leicht als Sklaverei deuten kann oder als einen Übergang in diese. Wie schon gesagt, Rigorosität des Staates auch gegen die Grundherren macht dies weitgehend wieder wett. Hing nun aber die Besteuerung von der Fläche des je Wirtschaftseinheit bebauten Bodens ab, so liegt nahe, daß der Verfall dieser Flächen von Indiktion zu Indiktion an sich wohl die Möglichkeit bot, sich den Verpflichtungen zu entziehen, wenn Eigentum nicht mehr profitabel oder angesichts der Belastungen durch die öffentliche Hand untragbar geworden war. Nach römischer Sklavenhalterpraxis - man lese bei Plutarch über Cato nach - bedeutet dies, 
der Grundherr brauchte nur seine Sklaven loszuschlagen und die Colonen zu entlassen, bzw. die Pachtverhältnisse aufzulösen, um sich seinen Pflichten zu entziehen. Die Menschen aber wurden dann, und dies besonders jetzt, in der späten Kaiserzeit, mittellos in eine Welt hineingestoßen, die von Katastrophen bestimmt war, ihnen weder materiell noch physisch große Aussichten für ein Überleben bot, und in der sie in Kürze untergehen mußten.

Dies aber nun ist die zweite Seite, die man gerne übersieht. Es ist wohl der Druck des Staates, der den Einzelnen an den Boden preßt, weil er ihn braucht. Derselbe Staat aber ist es, der diesem Einzelnen in solchen Zeitläuften zugleich eine Heimstatt zu geben sich bemüht, von der er nicht vertrieben werden kann, und damit das Menschenmögliche tut, ihm ein Existenzminimum zu sichern. Wie gesagt, rigorose Unterordnung, meinetwegen Ausbeutung, ist ein Aspekt der Sache. Fürsorge jedoch steht ihm wenigstens als ein Prinzip gleichwertig gegenüber, anders lassen sich die Bestimmungen nicht verstehen, man mag dabei partielle Verschiebungen unter einzelnen Kaisern registrieren, und dies hat, die allgemeinen Umstände lassen keine andere Deutung zu, neben allem Pragmatismus doch auch einen zutiefst sozialen Zug. Eine, wenngleich arme, aber doch in ihrer Weise feste Unterschicht, das war, trotz aller Bindungen, die diese auf sich zu nehmen hatte, das Beste, was sich noch erreichen ließ. Sicher, das Wort von einem Staatssozialismus ist eine Platitüde, hier angewendet, und, wie die meisten der scheinbaren Termini aus einer solchen Interpretationssphäre, besagt es im Grunde nichts. Rudimentäre Elemente eines solchen aber lassen die kaiserzeitlichen Gesetzestexte sehr wohl erkennen, und um sie kam man denn kaum herum, wollte man das Fortleben sichern. Gerne auch führt man die Wirksamkeit von Gedanken zur Ethik oder philosophischen Erkenntnissen an, um sich eine solche Entwicklung zu erklären, die sich noch vor der constantinischen Wende abzuzeichnen scheint. Sicher, eine Rolle mag all dies spielen, wie weit und wie tief solche Humanitätsvorstellungen gingen, indes läßt sich kaum sagen, man hat nicht den Eindruck, der Kreis der auf diese Weise Beeinflußten sei übergroß gewesen. Die pragmatischen Erwägungen, die um die eigene Existenz kreisten und die sich auf die Erhaltung der sozialen Struktur bezogen, müssen die Betroffenen stärker bewegt haben. Die gelegentlich aufscheinende Sentimentalität selbst unserer Gesetzestexte braucht dem nicht zu widersprechen, die erwähnte Rigorosität eines Constantin ist anders nicht zu verstehen.

Die Interpretation hat sich wohl allzu sehr daran gewöhnt, Freizügigkeit und Unabhängigkeit als unverrückbare Ideale zu feiern und sie zum Maßstab auch für soziale Verhältnisse und deren Beurteilung zu machen. Es könnte sein, daß indes die Forschung bereits an die Vorstufen eines Umdenkungsprozesses gelangt ist, von dem sie noch nicht viel ahnt. Überdies: Daß an anderer Stelle und wohl in der Mehrzahl aller einschlägigen Fälle die Dinge sich nach Wunsch und erfolgreich für die Betroffenen entwickelten, brauchte nicht erwähnt zu werden, und auch nicht, daß damit den domini eine Rolle zufiel, die ihren Platz im sozialen Gefüge kaum erstrebenswerter machte. Wohl haben wir jenen Typ des servus terrae. Er ist deswegen aber eben doch nicht servus domini, auch wenn mit dem Begriff des dominus selbst die Gesetzestexte nicht kleinlich sind, ja die Gesetze, daraufhin überprüft, lassen auch jetzt noch neben Verpflichtung zur Fürsorge das der bloßen Machtausübung weitgehend verschwinden. Bei aller Fragwürdigkeit der faktischen wie rechtlichen Stellung, eine Verwischung der Zustände wurde nicht angestrebt. Bodennutzung zu allgemeinen und besonders zu Steuerzwecken des Fiskus hat mit Vergewaltigung oder mit geförderter Böswilligkeit nichts zu tun, und auch 
die scheinbare Brücke zum mittelalterlichen Feudalismus kann, von hier aus gesehen, nur auf äußerlichen Voraussetzungen beruhen.

Die Synthese äußerlich zueinander passender Symptome aus verschiedener Wurzel aber ist allemal eine Simplifikation von besonders gefährlicher Tragweite, wenn es um Deutung historischer Konsequenzen und allgemeiner Entwicklungsformen geht, die sich zwangsläufig ergeben. Übrigens bleibt, genau geprüft, immer noch fraglich und das Gegenteil kaum ganz lückenlos zu beweisen, ob der Zwang in solcher Weise tatsächlich stets ganze Familien in Abhängigkeit brachte, die sich demnach verbreitete und so zugleich perpetuierte, und nicht lediglich mit den Kriterien der Erbrechtsbestimmungen beurteilt werden muß. Daß für den Staat die Abhängigkeit eine sekundäre, freilich notwendige Folge allgemeiner Zustände war, ist klar, denn nur dort, wo es um die juristischen Konsequenzen technischer Fragen und letztlich um die Erhaltung bestehender Zustände mithilfe der verfügbaren Arbeitskraft geht, ist von ihr die Rede. Ganz in Gegensatz etwa zu den germanischen Nachfolgestaaten scheint man zwischen Verbrechen und Wirtschaftsvergehen gerade auf dem Personalmarkt sehr wohl zu unterscheiden und urteilt dort milder, wo es sich etwa um bloße Abwerbung handelt, die öfters vorgekommen zu sein scheint und gelegentlich vielleicht die Gefahr einer eigenen Fluktuation in sich barg, die alles lähmte.

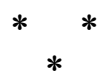

Gegen Ende des 4. Jhdts. scheinen sich denn auch die Folgen constantinischer Rigorosität einigermaßen abgeschliffen zu haben. Das heißt, man geht mit Standesfragen wieder großzügiger um und auch die Freilassung wird offensichtlich erleichtert. Auch die Kirche hatte denn, und dies nicht ohne eine offizielle Duldung, in der Zwischenzeit begonnen, in diesen Dingen eigene Wege zu gehen: Es sind dies freilich nicht die, die man sich bei einem oberflächlichen Blick auf eine christliche Soziallehre erwartet. Die Ethik, von der sie ausgehen und zu der sie zurückführen, ist eher von der pragmatischen, scheinbar vordergründigen, aber doch zeitlosen Erkenntnis bestimmt, daß eine gewaltsame, generelle Änderung bestehender Zustände unweigerlich in die Katastrophe führen müsse, die auch das eigene Anliegen mit betraf. Es ist jetzt schwerer als zuvor, aus den erhaltenen Nachrichten ein in sich geschlossenes Bild zu konstruieren, man hat den Eindruck, in der Gesetzgebung und entsprechender Selbstdarstellung nimmt nach Constantin eine gewisse Hektik zu, die sich nicht zuletzt wohl auch aus den Schwierigkeiten der Verarbeitung der neuen Postulate mit erklärt.

Die deutlich erschwerte allgemeine Lage kommt hinzu, die Frage, wieviel von dem Erhaltenen etwa dazu dient, den kaum mehr zu verbergenden Bruch zwischen Ost- und Westhälfte des Imperiums aufzuhalten oder in einer gewissen Autosuggestion zu kaschieren, ist kaum mehr zu beantworten. Um die Mitte des Jahrhunderts etwa sind christliche Sklaven jüdischer domini eo ipso frei, das kirchliche Asyl gewinnt an Wichtigkeit und bedeutet auch im Rechtlichen, wenn vielleicht auch nicht im Wirtschaftlichen, stets wohl Aufschub, Erleichterung, Fürsprache und Schutz vor den schlimmsten Repressalien. Eine Gefährdung der allgemeinen Stabilität etwa von Seiten der etablierten Kirche her scheint man aber nie befürchtet zu haben, eher im Gegenteil. Diese Kirche schafft denn die Sklaverei nicht ab, was auf den ersten Blick verwundern mag, und auch in Fragen der Freilassung hält man sich bewußt zurück. 
Indes, die äußere Stabilität ist die Voraussetzung für die innere, und die Verwirklichung einer sozialen Utopie konnte nicht die Absicht einer Soziallehre sein, die auf ein Reich vorbereiten sollte, das nicht von dieser Welt war. So haben denn weder ein Christus, ein Paulus noch die Kirchenväter der frühen Zeit das Sklavenproblem für wichtig genug gehalten, um Änderungen zu fordern, und auch Spätere halten sich daran. Hatte man eher davon auszugehen, daß es die bestehenden Verhältnisse waren, von denen aus man die eigenen Ziele am ehesten erreichte, so bleibt auch, gleichsam als Gebot, die soziale Lage nicht zu verändern. Bei all dem kann von einer solchen Voraussetzung aus auch die heilsgeschichtliche Einordnung kein großer Schritt gewesen sein, für die neben den Miseren, die alle betrafen, der Status unwichtig wird gegenüber der $\delta o v \lambda \varepsilon i ́ \alpha \tau \hat{\varphi} \theta \varepsilon \hat{\varphi}$.

Diese Einordnung nun mag sich in der Tat mit auf jene philosophischen, besonders die stoischen Traditionen auf der einen und einem Menschenbild auf der anderen Seite gründen, das vor Gott alle gleich macht oder nur graduelle Unterschiede kennt, für das aber die äußeren Bedingungen oder die gültigen Rechtsgrundlagen ohne Belang sind, auch wenn man sie keineswegs ignoriert.

Wohl ist in einer spirituellen Perspektive und deren Tradition dieser Sklave als ein Mensch, als ein Bruder gesehen. Doch für die religiöse Anthropologie, für die die Sklaverei allein im Unterworfensein unter Sünde, Laster und Triebhaftigkeit besteht und die Selbstbefreiung ihre metaphyischen Konsequenzen besitzt, ist die äußere Lage des Betroffenen gegenstandslos, ja sogar eine Möglichkeit der Bewährung. Die similitudo dei ist dazu ein flankierender weiterer Aspekt. So mögen die Kirchenväter nach wie vor von schweren Bedingungen sprechen, von Flucht und tiefer Not. Es bleibt nach einer christlichen Sozialisierung zu fragen, die dieses Neue in die Wirklichkeit des Imperiums einbezieht. Die kirchliche Auslegung ignoriert die entsprechenden allgemeinen, sozialen wie politischen Fragen eher, wie natürlich. Geht es aber um die Stabilität der Lebensmöglichkeiten, ohne die selbst eine christliche Welt weder sich auszubreiten noch überhaupt zu existieren vermag, so gewinnt denn im Gebot der caritas jene heilsgeschichtliche Deutung auch ihren saecularen Sinn. Freilich, in einer solchen Deutung tritt neben den servus bonus auch ein dominus mit ethischen Verpflichtungen von Sorge, Fürsorge, und letztlich einer christlichen Erziehung, Ausbildung fast im Sinne eines alten, patriarchalischen Ideals.

Eine pragmatische Komponente kommt hinzu. Die Freilassung aller Sklaven zwar hätte den Zusammenbruch nicht nur der Versorgung, sondern der gesamten Wirtschaft bedeutet. Die Diskussion um die Freiheit der Sklaven braucht dennoch unter den Kirchenvätern niemals aufzuhören. Sie konnte indes keine Forderung in sich bergen, die nach Verwirklichung drängte. So hat denn auch immer wieder die Mahnung der Kirchenväter ihr Gewicht, doch um Gotteswillen loyal und im Dienste den Herren gegenüber gehorsam zu bleiben. Es ist der Versuch, Bestehendes bestehen zu lassen, da der Rückweg zu jener alten patriarchalischen Struktur des Lebens verbaut war, auch wenn eine solche jetzt immer wieder idealisiert wird, dafür aber Gegensätze auszugleichen, Krisen zu verhindern und dabei die Hilfe der christlichen Religion dazu in Anspruch zu nehmen, um einen Zustand zu sichern, ohne den die Zukunft auch einer christlichen Welt auf Erden fraglich wird. Die Not freigelassener oder entlaufener Sklaven ohne Berufskenntnis, die in den Schriften der Kirchenväter gelegentlich anklingt, zeigt eine Frage auf, die die weltliche Seite, die Landbesitzer wie die öffentlichen Stellen, sehr wohl 
begriffen haben müssen. Für das Dilemma, wie der Fall eines Colonen oder Sklaven zu behandeln sei, der auf eine ungesetzliche Weise in den kirchlichen Dienst, zu einem Priester- oder gar Bischofsamt gelangt war, kam man zu einer allgemeinen, verbindlichen oder plausiblen Lösung naturgemäß nicht, daß es beide Seiten, die weltliche wie die kirchliche traf, liegt auf der Hand. Die Schroffheit eines Papstes wie Gelasius in der Behandlung solcher Fragen ist ein persönliches Bekenntnis über den kirchenrechtlichen Standpunkt hinaus, mochte im Osten ein Basilius auch zu Kompromissen eher bereit sein.

Der Colone wiederum, will man in ihm den rechtlich Freien sehen, behält seine vielfältigen Funktionen und die entsprechenden Bezeichnungen. Verläßt er seinen Platz, ist er zurückzuholen, doch wird jetzt betont, daß er nach 30 Jahren bleiben könne, wo er sei. Die Verordnung in einem analogen $\mathrm{Zu}-$ sammenhang ist alt und wurde in der Antike mehrfach verwendet, auch an Erörterungen fehlt es nicht, und in der Tat, sie läßt sich für oder wider die Betroffenen deuten. Ich möchte indes glauben, gerade jetzt habe die betonte Kontrolle ihren Sinn, weil neben der allgemeinen besonders seine eigene wirtschaftliche Sicherung wichtig war, die nur auf diese Weise garantiert werden konnte. Immerhin, er darf selbständig Handel betreiben und eigene Initiative auf diesem Sektor entwickeln, wenn er dadurch nur nicht ganz zum Kaufmann wird. Und wie gesagt, es gibt das Gebot, daß censiti, Personen, die innerhalb einer Wirtschaftseinheit versteuert sind, nicht veräußert werden dürfen. Bereits Constantin spricht in einem Gesetz (CTh 2, 25, 1) für den Fall der Besitzteilung davon, es sei unmenschlich, bei solcher Gelegenheit Einzelne von ihren Familien zu trennen. Die Frage nach dem Status der Betroffenen ist bei all dem so wenig zu stellen wie die nach der Art ihrer wirtschaftlichen Abhängigkeit.

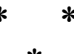

So stellt denn das Imperium demnach einen Fleckenteppich von Rechtsbeziehungen, Verwaltungseinheiten und Wirtschaftskörpern mit vielfältigen regionalen Unterschieden und von sozialen Gegebenheiten nebeneinander dar. Und es ist schwer vorzustellen, daß ein mit einschlägigen Problemen Befaßter noch die nötige Durchsicht besaß, was denn alle Entscheidungen von vornherein zum Provisorium werden ließ, von den Möglichkeiten der Kommunikation zu schweigen, die die entsprechenden Erlasse überhaupt an die zuständigen Stellen brachte. Neben der Beamtenschaft und dem Heer bedeuten die Grundbesitzer eine dritte Kraft, ohne die die anderen beiden nicht denkbar sind. Daß der Staat sie, von außen gesehen, weniger beeinträchtigt als die anderen Gruppen, liegt in der Natur der Sache, ihre Exklusivität, zu beobachten in Rom oder im Gallien der späten Antike, aber scheint Selbsttäuschung, denn es ist, wie gezeigt, allein die Lastenverteilung und die Einsparung weiterer, schließlich auch staatlicher Kräfte und Institutionen, die gerade durch sie ermöglicht wird, was offiziell diese Sonderstellung und später auch deren Bewahrung unter germanischer Herkunft erklärt. Sicher, diese Gruppe hebt sich von den unteren Schichten äußerlich ab, Übergriffe mag es geben, die eine gewisse Unüberbrückbarkeit von Differenzen auch in den Interessen zeigen. Die Klasse als Ganzes aber als eine Ausbeutermaffia mit dem Ziel eines permanenten Bauernlegens mit zu klassifizieren, wie es etwa Salvian tut, ist Schwarzmalerei. Wie angedeutet, es gab auch für sie Gründe, den Bogen in diesen Dingen nicht zu überspannen. Und das gleiche gilt von dem Bilde eines Klassenkampfes, der über ein Jahrhundert seine merkwürdige 
Rolle eines ideologischen Opiates auch noch in den eben vergangenen Epochen spielte. Was aber die Exklusivität betrifft - die spätantike Prosopographie weist genug an Beispielen auf, wie diese Schicht Neulinge aus niederen Rängen der Gesellschaft, ja barbarischen Ursprungs, aufzunehmen hatte. Wie bekannt, sind denn die Urgroßväter der großen, spektakulären spätantiken Senatoren in Rom mit ihrem Reichtum, ihren Ansprüchen und auch mit ihrer allzu sichtbaren Verkörperung altrömischer Aristokratie, zum großen Teil noch Sklaven oder Freigelassene gewesen. Was immer man von diesem Kreis nicht immer erfreulicher Persönlichkeiten hält, die Möglichkeiten sozialer Mobilität sollte man nicht übersehen, die offensichtlich die Kaiserzeit hindurch bestanden.

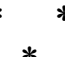

All dies freilich hat die Krise, die 376 begann, nicht aufhalten können. Auch sie schließlich hat als Anlaß eine finanz- und bevölkerungspolitische Absicht des Kaisers Valens, der durch die leicht zu gewinnenden Germanen die Stellung von neuen Truppen aus dem Imperium oder einen Zwang zu erhöhter Geldzahlung erspart wissen wollte. Diese Absicht, wie angedeutet, steht im Rahmen einer jahrhundertealten Tradition, die Folgen zu übersehen waren diesmal weder der Kaiser in der Lage noch seine Ratgeber. Wie bekannt, ging in den Jahrzehnten danach der Westen verloren, der Osten konnte sich durch eine Einigungsbewegung und eine Konzentration größten Ausmaßes noch über ein Jahrtausend halten.

So bleibt am Schlusse noch zu fragen, ob neben erwähnten defensiven Versuchen das Imperium denn nichts Offensives unternahm, seine Schwäche von innen heraus zu kompensieren. Vielleicht müßte man von hier aus die Entwicklung römischer Militärpolitik dieser letzten Zeit noch einmal überprüfen. Die Rolle einer Berufsarmee als Element der sozialen Stabilisierung scheint denn seit ihrer Genese unter Marius unverkennbar, mag spätere Deutung auch die eher gefährliche Komponente des Phänomens betonen. Die Entwicklung des Veteranenproblems indes ist nicht anders zu verstehen, und die Privilegien für Einzelne hatten stets wohl ein Ziel im Hintergrund, das über den Bereich bloßer Militärpolitik hinausweist. Zwar ist ein Zwiespalt zwischen Heer und ziviler Welt, zwischen Verteidigung und Wirtschaft seit Diokletian geblieben. Es paßt indes in unseren Rahmen, wenn wir nun verstärkt etwa von militärischer Selbstversorgung wenigstens stationierter Truppen erfahren; und selbst wenn dabei vor allem eine eigenartige frontier society etwa in den Grenzgarnisonen entstand, die versuchte und zum Teil verwirklichte Entlastung der Wirtschaft dabei ist nicht zu übersehen. In der späten Antike ist dies noch mehr Selbstverständlichkeit. Der aktive Soldat, der neben seinem Dienst noch einem Gewerbe nachgeht, begegnet in Ägypten und anderswo, und daß man ihn duldet, hat seine Gründe, auch wenn dies auf Kosten der militärischen Effizienz gehen und den unmittelbaren Vorgesetzten mitunter Schwierigkeiten bereiten mag: Ein Zeugnis wie etwa die Abinnaeuspapyri steht für vieles andere. Offensichtlich tat man seit je alles, mittels gezielter Entlassungsmethoden die überstrapazierte Wirtschafts- und Sozialstruktur zu verbessern. Ein Netz von Veteranenkolonien seit dem Beginn des Prinzipats durchzog das ganze Reich, muß sich mit der Zeit durch eine Selbstergänzung verdichtet und regeneriert haben und ließ sich bei allen bekannten Vorrechten und Immunitäten für Einzelne in das allgemeine Gefüge eingliedern. Und wir haben Zeugnisse in Fülle, daß dies auch für die aufgenomme- 
nen Barbaren versucht wurde, wie weit immer sie sich integrieren ließen, was eine Romanisierung bedeutete. Ihre Vermehrungsfreudigkeit registriert ein Ammian mit Staunen, und es gibt Zeugnisse dafür, daß man sie einzeln oder in Gruppen auch in der Spätantike noch gerne aufnahm, wenn die Lücken im Gefüge der Imperiumsbevölkerung allzu groß wurden. Die Rolle der Armee als Wirtschaftsfaktor ist alt, und sie muß mit der Einrichtung von festen Garnisonen neue Aspekte gewonnen haben. Neben der Romanisierung ihrer Mitglieder und der sozialen Funktion der Veteranen, die man zweifellos früh bereits einkalkulierte, steht die Kultivierung von Grenz- wie Provinzgebieten von Anfang an, neben dem Einstieg Einzelner aus diesem Kreise selbst in die Geldwirtschaft, wenngleich wohl in bescheideneren Dimensionen, und allgemein die erwähnte Ansiedlung nach dem Ende des Dienstes als allgemein stabilisierender Faktor. Zeugnisse für eine Vorliebe von Imperiumsbewohnern für den Militärdienst fehlen nicht. Doch es ist zu vermuten, daß diese für den zivilen Bereich und die Wirtschaft immer mehr unabkömmlich und höchstens zur Bildung von Truppenkadern noch eine Funktion besaßen. Daß mit den Katastrophen des 3. Jhdts. diese Armee zu einer steuerlichen Belastung wurde, liegt auf der Hand. Aber unverkennbar gehen mit der Barbarenaufnahme, mit Laetenansiedlung, Deditizierbehandlung und Gentileneingliederung die Bemühungen um eine Selbstversorgung und damit um steuerliche Entlastung der zivilen Bevölkerung einher, wofür seit dem 3. Jhdt. die Einrichtung des autonomen Grenzheeres eine Vorstufe sein wird, die man auf lange Sicht hin kalkulierte. Für diese neue Armee spielt der Rechtsstatus des Einzelnen keine Rolle mehr, doch für die Integration Fremder, selbst von Barbaren, hatte man längst praktikable Modalitäten entwickelt. Am Ende der Völkerwanderung, in Italien, in Gallien, scheint in der Tat ein neuer Modus erreicht, das Problem, wenngleich jetzt unter allgemeinen Bedingungen, zu lösen.

So hat denn wohl auch jenes Hospitalitätsgesetz 398, das diesen Fremden ein Drittel jeweiligen Landes sichert, seinen wirtschaftspolitischen Hintergrund, von der Ansiedlung ganzer Gefangenenstämme 409 spricht CTh 5, 6, 3. Kein Wunder, daß man sich unablässig bemüht, die entsprechenden Möglichkeiten zu klären oder Kategorien zu definieren. Es geschieht nicht ohne eine Zielsetzung auf längere Sicht und auch nicht ohne einen eher vordergründigen Opportunismus, wenn die Kaiser viele Generationen hindurch alles versuchen, um den so gewonnenen Kräften und ihrem Anhang etwas wie eine Heimat zu geben, während sie sich in deren Dienst verzehren. Die soziale neben der rein wirtschaftlichen Komponente läßt sich nicht einmal hier übersehen, doch zugleich wurde auf diese Weise die Bildung der Nachfolgestaaten gefördert. Und selbst ein Attila muß sich in diesem Rahmen gesehen haben.

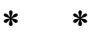

*

Wir sind damit am Ende. Was anzudeuten war, ist, daß die Entwicklung innerhalb des Imperiums in unserem Bereich keineswegs das Spiel blinder Kräfte war, die es schließlich in die Katastrophe hineintaumeln ließen. Das Imperium ist sicher kein moderner Staat. Er mochte auf Erfahrungen und Vorbilder zurückgreifen, aber von einem erkannten Fortschritt, das bedeutet unter solchen Umständen, von der Wertigkeit sozialer Problematik in einem modernen Sinne ist für die Zeit, um die es hier geht, d. h. für den Anfang, nicht zu reden. Indes - die Kaiserzeit, besser gesagt, die Monarchie, die dazu am ehesten die Voraussetzung in sich birgt, scheint früh zu lernen, und späte- 
stens seit dem 2. Jhdt. deutet sich eine neue Problemstellung an. Und je brennender die Notwendigkeit der Fortexistenz sich zeigt, desto wichtiger wird der neu entdeckte Bereich, wird die Entdeckung des Wechselverhältnisses zwischen beiden, der wirtschaftlichen und der sozialen Frage überhaupt, und die Erkenntnis, daß es ohne ihre Verbindung kein Fortbestehen des Imperiums gibt.

So zwingt wirtschaftliche Notwendigkeit zu einer Veränderung innerhalb der unteren Schichten wie auch des Verhältnisses zu ihnen, es kommt zu Ansätzen einer rational und reflektiert herbeigeführten Änderung jener typisch antiken Bevölkerungsstruktur und zu einer Teilung in zwei Gruppen, deren unterschiedliche rechtliche Behandlung naturgemäß doch nur eine oberflächliche war. All dies freilich bleibt Versuch, und zu einer umfassenden Neuordnung des gesamten Gefüges kam es nicht, mochten sich die entsprechenden Kenntnisse von dem, was nötig war, auch verfestigen. Auch an gutem Willen fehlte es nicht, wie die Ansätze zeigen. Die spätere Entwicklung, von außen aufgezwungen, läßt den Prozeß scheinbar wieder abbrechen. Genau besehen aber, ein Ende findet er nicht, er würde hier, weiter verfolgt, etwa für den frühbyzantinischen Staat andeuten, daß die Entwicklung keineswegs abbrach, auch wenn neue Bedingungen Veränderungen erzwangen, die an einer Kontinuität zweifeln lassen.

Das Bemühen, wenigstens die Grundlagen der Wirtschaft stabil zu halten, bewirkt denn gegen das Ende der Antike hin den Übergang zu einer Gesellschaft, die nicht nur in philosophischer Selbstdeutung, sondern auch in Wirklichkeit nur noch zweierlei Menschen kennt, aufeinander angewiesen und im besten Sinne des Wortes einander verpflichtet, den Herrscher und den Untertan.

\section{Sachdienliche Literatur in Auswahl}

Abbot, F.; Johnson A. Municipal administration in the Roman empire, Princeton 1926

Alföldy, G.

Alföldy, G.

Alföldy, G.

Römische Sozialgeschichte ${ }^{3}$, Wiesbaden 1984

Die römische Gesellschaft, Stuttgart 1986

The crisis of the 3rd century as seen from contemporaries, GRBS 15, 1979, $89 \mathrm{ff}$.

Alföldy, G.

Die Krise des römischen Reiches, Stuttgart 1989

Alföldy, G.; Dobson, B.; Eck, W. (Hg.), Kaiser, Heer und Gesellschaft in der römischen Kaiserzeit, Stuttgart 2000

Andersen, Th.

Patrocinium. The concept of personal dependencies in the Later Roman empire and the early Middle-Ages, New York 1974

Arnheim, M. T.

The senatorial aristocracy under the later Roman Empire, Oxford 1972

Barnes, T. D.

Barnish, S.

Patricians under Valentinian III., Phoenix 29, 1975, $155 \mathrm{ff}$. Taxation and barbarian settlement in the Western Empire, PBSR 54, 1986, $170 \mathrm{ff}$.

Barnish, S.

Pigs, plebeians and potentes. Rome's economic hinterland, PBSR 55, 1987, $157 \mathrm{ff}$.

Bellen, $\mathrm{H}$.

Bellen, H. Studien zur Sklavenflucht im römischen Kaiserreich, Wiesbaden 1976

Bernardi, A.

Politik - Recht - Gesellschaft. Studien zur Alten Geschichte (hg. v. L. Schumacher), Stuttgart 1997

The economic problems of the Roman Empire at the time of its decline, SDHI 31, 1965, $110 \mathrm{ff}$.

Bieczunska-Malowist, I. L'esclavage dans l'Égypt gréco-romain, Warschau 1977 
Bleckmann, B.

Bleicken, J.

Boak, A.

Bolkestein, H. Bracher, K. D.

Brockmeyer, N.

Brockmeyer, N.

Brunt, P. A. (Hg.)

Burns, T. S.

Carl, G.

Chauvot, A.

Chianea, G.

Christ, K. (Hg.)

Clausing, R.

Cracco-Ruggini, L.

de Ste. Croix, G.

Czuth, B.

Demandt, A.

Dohr, H.

Drinkwater, J.; Elton, H. (Hg.), 5th century Gaule. A crisis of identity?, Cambridge 1992 Durliat, J.

Durliat, J.

Eck, W.; Wolff, H. (Hg.)

Eck, W.; Heinrichs, J.

Eibach, D.

Finley, M. (Hsg.)

Fischer, J.

Fustel de Colanges, N.

Garnsey, Ph.

Garnsey, Ph. bridge 1988
Gellner, E.; Waterbury, J. (Hg.), Patrons and clients in mediterranean societies, London

1977

Goffart, W.

Goffart, W.

Goffart, W.

Grieser, H.

Die Reichskrise des 3. Jhdts. in der spätantiken und byzantinischen Geschichtsschreibung, München 1992

Verfassung und Sozialgeschichte des römischen Kaiserreiches, Paderborn 1978

Manpower shortage and the fall of the Roman empire in the West, Ann Arbor 1955

De colonatu Romano eiusque origine, Diss. Amsterdam 1906 Verfall und Fortschritt im Denken der frühen römischen Kaiserzeit, Köln 1987

Arbeitsorganisation und ökonomisches Denken in der Gutswirtschaft des römischen Reiches, Diss. Bochum 1966

Sozialgeschichte der Antike, Stuttgart 1972

The Roman Economy, Oxford 1974

Barbarians within the gates of Rome. A study of the Roman military policy and the barbarians ca. 375-425 a. D., Bloomington 1994

Die Agrarlehre Columellas in soziologischer Betrachtung, Diss. Heidelberg 1925

Opinions romaines face aux barbares de IV s. apr. J. C., Paris

Les idées politiques de Sidoine Apollinaire, RD 47, 1969, $359 \mathrm{ff}$.

Der Untergang des römischen Reiches, Darmstadt 1970

The Roman Colonate, New York 1925

Economia e società nell'Italia annonaria² ${ }^{2}$, Bari 1995

The Class Struggle in the ancient world, London 1981

Die Quellen der Geschichte der Bagauden, Szeged 1965

Der Fall Roms, München 1984

Die italischen Gutshöfe nach den Schriften Catos und De la ville antique à la ville byzantine. Le probléme des subsistances, Rom 1999

Les rentiers de l'import. Recherches sur les finances municipales dans la pars orientis au IV. s., Wien 1993

Heer und Integrationspolitik. Die römischen Militärdiplome als Geschichtsquellen, Köln 1986

Sklaven und Freigelassene in der Gesellschaft der Kaiserzeit, Darmstadt 1993

Untersuchungen zum römischen Kolonat in der kaiserlichen Gesetzgebung unter bes. Berücksichtigung der Terminologie, Diss. Köln 1977

Studies in ancient society, London 1975

Die Völkerwanderung im Urteil der zeitgenössischen kirchlichen Schriftsteller Galliens unter Einbeziehung des Heiligen Augustinus, Heidelberg 1948

Les origines du systèm féodal. Le bénéfice et le patronat, Paris $\mathbf{1 8 9 0}$

Social status and legal privilege in the Roman Empire, Oxford 1970

Famine and food - supply in the greco-roman world, Cam-

Caput and colonate. Towards a history of Late Rome taxation, Toronto

Barbarians and Romans a. D. 418-584. The techniques of accomodation, Princeton 1980

Rom's fall and after, London 1989

Sklaverei im spätantiken und frühmittelalterlichen Gallien (5.-7. Jh.), Stuttgart 1997 
Grünewald, Th.

Gummerus, H.

Herrmann, J.

Hermann, E.

Hermann, E. (Hermann-Otto), Ex ancilla natus. Untersuchungen zu den hausgeborenen Sklaven und Sklavinnen im Westen des römischen Kaiserreiches, Stuttgart 1994

Herz, P.

Horstkotte, H.

Johne, K. P. (Hg.)

Jones, A. H. M.

Jones, A. H. M.

Jones, A. H. M.

Jones, A. H. M.

Karayannopoulos, J.

Kiechle, F.

Klein, R.

Klein, $\mathrm{R}$.

Kohns, H. P

Kolendo, J.

Kontoulis, G.

Krause, J. U.

Krause, J. U. u. a. (Hg.)

Krumeich, Chr.

Krumpholz, H.

Ladner, G.

Langhammer, W.

Laub, F.

Latouche, R.

Liebenam, W.

Liebeschuetz, W.

Löhken, H.

Lot, F.

Matthews, J.

MacMullen, R.

MacMullen, R.

MacMullen, $\mathrm{R}$.

Räuber, Rebellen, Rivalen, Rächer. Studien zu den latrones im römischen Reich, Stuttgart 1999

Der römische Gutsbetrieb als wirtschaftliche Organisation in den Werken des Cato, Varro und des Columella, Leipzig 1906

Kleine Schriften zur Rechtsgeschichte, München 1990

Ecclesia in re publica, Stuttgart 1980

Studien zur römischen Wirtschaftsgesetzgebung. Die Lebensmittelversorgung, Stuttgart 1988

Die Theorie vom spätrömischen Zwangsstaat und das Problem der Steuerhaftung, Königstein 1984

Gesellschaft und Wirtschaft des römischen Reiches im 4. Jhdt., Berlin 1993

The roman colonate, P\&P 13, 1958, $1 \mathrm{ff}$.

The Later Roman Empire, Oxford 1964

The decline of the ancient World, London 1966

The Roman economy, Oxford 1974

Das Finanzwesen des frühbyzantinischen Staates, München 1958

Sklavenwirtschaft und technischer Fortschritt, Wiesbaden 1969

Die Sklaverei in der Sicht der Bischöfe Ambrosius und Augustinus, Stuttgart 1988

Die Haltung der kappadokischen Bischöfe Basilius von Caesarea, Gregor von Nazianz und Gregor von Nyssa zur Sklaverei, Stuttgart 2000

Versorgungskrisen und Hungerrevolten im spätantiken Rom, Diss. Bonn 1961

Le colonat en Afrique sous le haut-empire, Paris 1976

Zum Problem der Sklaverei bei den kappadokischen Kirchenvätern und Johannes Chrysostomus, Bonn 1993

Spätantike Patronatsformen im Westen des römischen Reiches, München 1987

Schichten, Konflikte, religiöse Gruppen, materielle Kultur. Bibliographie zur römischen Sozialgeschichte, Stuttgart $1994 \mathrm{ff}$.

Hieronymus und die christlichen feminae clarissimae, Diss. Bonn 1993

Über sozialgeschichtliche Aspekte in der Novellengesetzgebung Justinians, Diss. Bonn 1992

On Roman attitudes toward barbarians in the later Antiquity, Viator 7, 1976, $1 \mathrm{ff}$.

Die rechtliche und soziale Stellung der magistratus municipales und der Decurionen, Wiesbaden 1973

Die Begegnung des frühen Christentums mit der antiken Sklaverei, Stuttgart 1982

Les origines de l'économie occidentale (IV-IX s.), Paris 1956 Stadtverwaltung in römischer Kaiserzeit, Leipzig 1900

Bishops and barbarians, Oxford 1990

Ordines dignitatum. Untersuchungen zur formalen Konstituierung der spätantiken Führungsschicht, Köln 1982

L'impôt foncier et la capitation personelle sous le bas-empire et à l'époque franque, Paris 1928

The Roman Empire of Ammianus, London 1989

Soldier and civilian in the Later Roman Empire, Cambridge Mass. 1963

Roman Government's response to crisis a. D. 235-337, New Haven 1976

Late Roman slavery, Historia 36, 1987, $359 \mathrm{ff}$. 
de Martino, F.

Millar, F.

Mirkovic, M.

Müller-Mertens, E. (Hg.) Feudalismus. Entstehung und Wesen, Berlin 1985

Noethlichs, K. L.

Oehme, M.

Patlagean, E.

Pohl, W. (Hg.)

Quast, F.

.

Reduzzi Merola, F. Stordi Mar

Reduzzi Merola, F.; Stordi Marina, A. (Hg.), Femmes-esclaves. Modèles d'interpretation

Riccardi, S. anthropologique, économique, juridique, Neapel 1999

Rostovtzeff, M.

Rostovtzeff, M.

Rouland, N.

Salmon, P.

Samson, R.

Schnappinger, I.

Schulten, A.

Schumacher, L.

Seipel, I.

Seyfarth, W.

Shaw, B.

Stahl, M.

Stroheker, K. F.

Tinnefeld, F.

Treucker, B.

Unruh, F

Vogt, J.

Wacke, A.

Waldstein, W.

Weber, M.

Weber, M.

Storia economica di Roma antica, Firenze 1979 (dt. v. Brigitte Galsterer, Wirtschaftsgeschichte des alten Rom, München 1985)

Empire and city. Augustus to Julian. Obligations, excuses and status, JRS 73, 1983, $76 \mathrm{ff}$.

The colonate liberty and Justinian's humanity,

baden 1981

Die römische Villenwirtschaft. Untersuchungen zu den Agrarschriften Catos und Columellas und ihrer Darstellung bei Niebuhr und Mommsen, Bonn 1988

Pauvreté économique et pauvreté à Byzance IV.-VII. s., Paris 1977

Kingdoms of the Empire. The integration of barbarians in late antiquity, Leiden 1997

Die Honoratiorenschicht in den Städten des griechischen Ostens. Untersuchungen zur politischen und sozialen Entwicklung in hellenistischer und römischer Zeit, Stuttgart Die Erforschung der antiken Sklaverei in Italien vom Risorgimento bis Ettore Ciccotti, Stuttgart 1997

Studien zur Geschichte des römischen Kolonats, Leipzig 1910

Social and economic history of the Roman Empire 2 , Oxford 1957

Pouvoir politique et dépendence pesonelle dans l'antiquité romaine, Brussel 1979

Population et dépopulation dans l'empire Romain, Brüssel 1974

Rural slavery, inscriptions, archaeology and Marx. A response to Ramsay Mac Mullen's Later Roman Slavery, Historia $38,1987,99 \mathrm{ff}$.

Die Gewerbesteuer in der Spätantike, Diss. München 1980

Die römischen Grundherrschaften, Weimar 1896

Servus Index. Sklavenverhör und Sklavenanzeige im republikanischen und kaiserzeitlichen Rom, Stuttgart 1993

Die wirtschaftlichen Lehren der Kirchenväter, Wien 1907

Soziale Fragen der spätrömischen Kaiserzeit im Siegel des Theodosianus, Berlin 1963

Bandits in the Roman Empire, P\&P 105, 1984, $3 \mathrm{ff}$.

Imperiale Herrschaft und provinziale Stadt, Göttingen 1978

Der senatorische Adel im spätantiken Gallien, Tübingen

1948

Die frühbyzantinische Gesellschaft, München 1977

Politische und sozialgeschichtliche Studien zu den Basiliusbriefen, Diss. Frankfurt 1960

Das Bild des Imperium Romanum im Spiegel der Literatur an der Wende von 2. zum 3. Jh. n. Chr., Bonn 1991

Sklaverei und Humanität. Studien zur antiken Sklaverei und ihrer Erforschung ${ }^{2}$, Stuttgart 1983

Die potentiores in den Rechtsquellen, ANRW II 13, 1980, $562 \mathrm{ff}$.

Operae libertorum. Untersuchungen zur Dienstpflicht freigelassener Sklaven, Stuttgart 1986

Römische Agrargeschichte, Stuttgart 1891

Agrarverhältnisse im Altertum: Ges. Aufsätze zur Sozial- und Wirtschaftsgeschichte, Tübingen 1924, 1 ff. 
Welwei, K.

Welwei, K.

Wallace, S.

Walser, G.; Pekary, Th. Waltzing, J.

White, K. D.

Wierschowski, L.

de Zulueta, F.
Unfreie im antiken Kriegsdienst, I Stuttgart 1973; II 1977; II 1988

Zur Ansiedlungspolitik Mark Aurels, BJ 186, 1986, $285 \mathrm{ff}$. Taxation in Egypt form Augustus to Diocletian, Princeton 1938

Die Krise des römischen Reiches, Berlin 1962

Étude historique sur les corporations professionelles chez les Romains depuis les origines jusqu'à la chute de l'empire d'occident, Louvain 1895

Roman farming, Ithaca 1970

Heer und Wirtschaft. Das römische Heer in der Prinzipatszeit als Wirtschaftsfaktor, Bonn 1984

De patrociniis vicorum. Oxford Studies in Social and Legal History 1, 1909, $1 \mathrm{ff}$. 